\title{
Chemical Diversity of Soft Coral Steroids and Their Pharmacological Activities
}

\author{
Ekaterina V. Ermolenko ${ }^{1}$, Andrey B. Imbs ${ }^{1}$, Tatyana A. Gloriozova ${ }^{2}$, Vladimir V. Poroikov ${ }^{2}$ (D), \\ Tatyana V. Sikorskaya ${ }^{1}\left(\mathbb{D}\right.$ and Valery M. Dembitsky ${ }^{1,3, *}$ \\ 1 A.V. Zhirmunsky National Scientific Center of Marine Biology, 17 Palchevsky Str., 690041 Vladivostok, \\ Russia; ecrire_711@mail.ru (E.V.E.); andrey_imbs@hotmail.com (A.B.I.); Miss.tatyanna@yandex.ru (T.V.S.) \\ 2 Institute of Biomedical Chemistry, bldg. 8, 10 Pogodinskaya Str., 119121 Moscow, Russia; \\ tatyana.gloriozova@ibmc.msk.ru (T.A.G.); vladimir.poroikov@ibmc.msk.ru (V.V.P.) \\ 3 Centre for Applied Research, Innovation and Entrepreneurship, Lethbridge College, 3000 College Drive \\ South, Lethbridge, AB T1K 1L6, Canada \\ * Correspondence: valery.dembitsky@lethbridgecollege.ca
}

Received: 20 October 2020; Accepted: 26 November 2020; Published: 2 December 2020

\begin{abstract}
The review is devoted to the chemical diversity of steroids produced by soft corals and their determined and potential activities. There are about 200 steroids that belong to different types of steroids such as secosteroids, spirosteroids, epoxy- and peroxy-steroids, steroid glycosides, halogenated steroids, polyoxygenated steroids and steroids containing sulfur or nitrogen heteroatoms. Of greatest interest is the pharmacological activity of these steroids. More than 40 steroids exhibit antitumor and related activity with a confidence level of over 90 percent. A group of 32 steroids shows anti-hypercholesterolemic activity with over 90 percent confidence. Ten steroids exhibit anti-inflammatory activity and 20 steroids can be classified as respiratory analeptic drugs. Several steroids exhibit rather rare and very specific activities. Steroids exhibit anti-osteoporotic properties and can be used to treat osteoporosis, as well as have strong anti-eczemic and anti-psoriatic properties and antispasmodic properties. Thus, this review is probably the first and exclusive to present the known as well as the potential pharmacological activities of 200 marine steroids.
\end{abstract}

Keywords: soft corals; steroids; chemical diversity; antitumor; anti-inflammatory; anti-eczemic; anti-psoriatic; biological activity prediction; PASS

\section{Introduction}

Soft corals belong to the order of Alcyonacea (class Octocorallia, Anthozoa, Cnidaria), formerly known as gorgonians $[1,2]$ living in all the oceans of the world, especially in the tropics and subtropics. In addition, photosynthetic corals are successfully cultivated in artificial conditions and are the subject of chemical and biomedical investigation [3,4]. Soft corals produce more than 5800 secondary metabolites [5], including rare and unusual fatty acids [6-13], terpenoids [14-16], quinones [17], alkaloids [18-23], glycosides [24-31] and steroids [18,32-37]. It is known that many secondary metabolites isolated from marine invertebrates including soft corals, exhibit anticancer and other pharmacological activities [38-45].

In this review, we will look at rare and unusual steroids isolated from soft corals belonging to the order of Alcyonacea. The biological activity of many steroids has not been determined and we present the pharmacological activities detected experimentally and predicted based on the structure-activity relationships using the PASS (Prediction of Activity Spectra for Substances) software. PASS estimates the probabilities of several thousand biological activities with an average accuracy of about $96 \%$. Probability of belonging to the class of "actives" Pa is calculated for each activity, providing the assessment of the hidden pharmacological potential of the investigated soft coral steroids [42]. 


\section{Steroids Derived from the Genus Sinularia}

Sinularia is a specific group of soft octocorals, a genus belonging to the family Alcyoniidae (class Anthozoa, phylum Cnidaria) and it includes about 175 actual species, 47 of which have been described only in the last quarter of a century $[46,47]$. Sinularia species are the most abundant corals in the entire Indo-Pacific region, especially in shallow water and dominate reef substrates [47].

Nowadays, many species of coral of the genus Sinularia are well adapted and cultured for biological and medical research and they are also an excellent source of many biologically active metabolites, including diterpenoids, unusual steroids and triterpenoids $[15,19,38,40,48,49]$.

Three steroids, ergosta-5,24(28)-diene-3 $\beta, 4 \alpha$-diol (1), 24(S),28-epoxyergost-5-ene-3 $\beta, 4 \alpha$-diol (2) and dissesterol (3) were found in the methanol extract of the Vietnamese soft coral Sinularia nanolobata. Compound (2) exhibited moderate cytotoxicity against acute leukemia (HL-60) cell line with $\mathrm{IC}_{50}$ value of $33.5 \mu \mathrm{M}$ and a weak effect on the hepatoma cancer (HepG2) and colon adenocarcinoma (SW480) cell lines with $\mathrm{IC}_{50}$ values of 64.3 and $71.0 \mu \mathrm{M}$, respectively [50]. The structures of the steroids (1-18) are shown in Figure 1 and the potential biological activities are shown in Table 1.
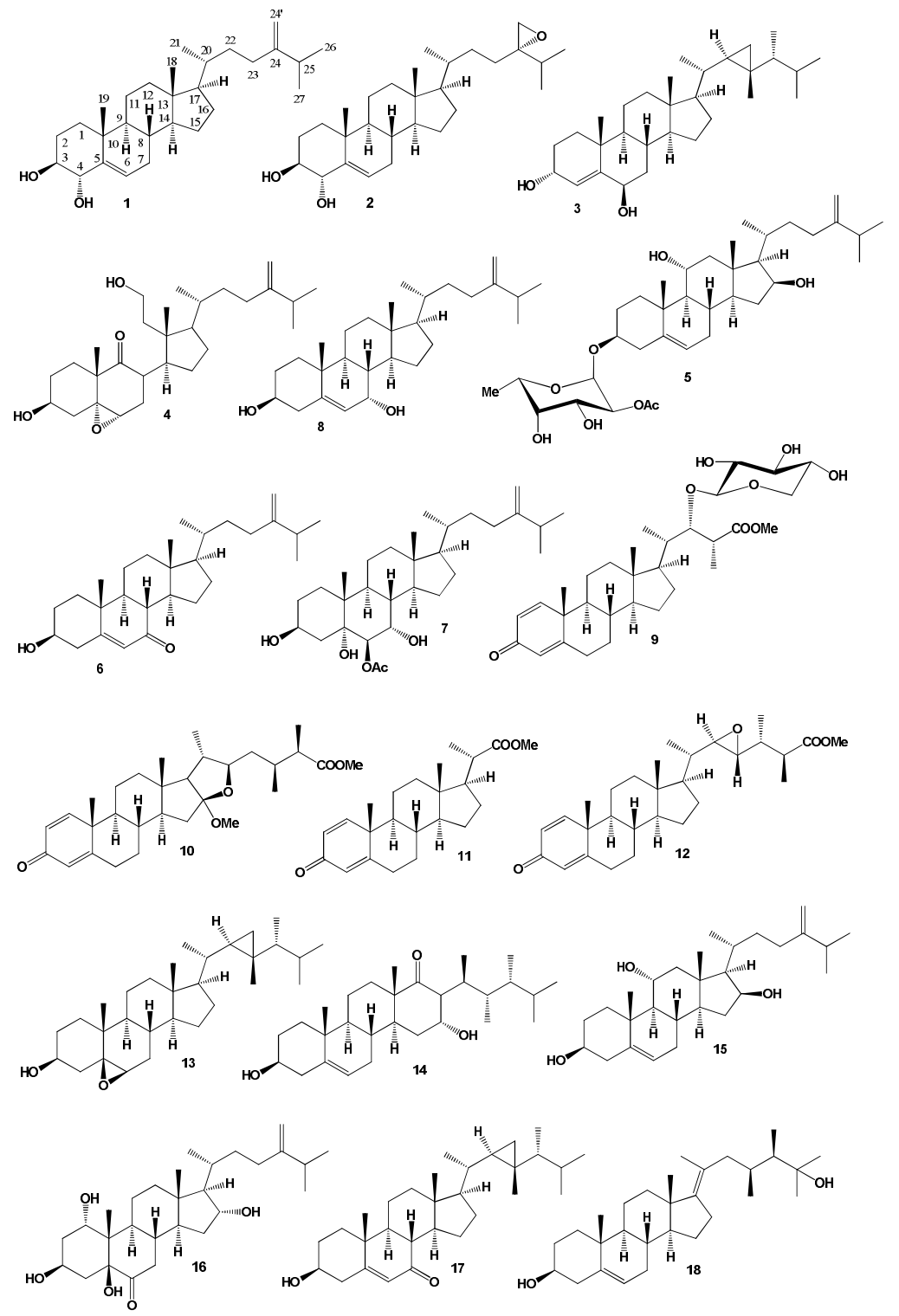

Figure 1. Bioactive steroids derived from the genus Sinularia. 
Table 1. Probable biological activities of steroids derived from the genus Sinularia estimated by PASS

(Prediction of Activity Spectra for Substances).

\begin{tabular}{|c|c|c|c|}
\hline No. & Antitumor and Related Activity, $(\mathrm{Pa})$ * & Lipid Metabolism Regulators, $(\mathbf{P a}) *$ & Additional Biological Activity, (Pa) * \\
\hline 1 & $\begin{array}{c}\text { Antineoplastic }(0.840) \\
\text { Chemopreventive }(0.806) \\
\text { Proliferative diseases treatment }(0.705) \\
\text { Antimetastatic }(0.562) \\
\text { Anticarcinogenic }(0.542)\end{array}$ & $\begin{array}{c}\text { Anti-hypercholesterolemic }(0.920) \\
\text { Hypolipemic }(0.798) \\
\text { Atherosclerosis treatment }(0.637) \\
\text { Hyperparathyroidism treatment }(0.523)\end{array}$ & $\begin{array}{c}\text { Respiratory analeptic }(0.879) \\
\text { Anesthetic general }(0.851) \\
\text { Antifungal }(0.792) \\
\text { Anti-inflammatory }(0.754) \\
\text { Antibacterial }(0.545)\end{array}$ \\
\hline 2 & $\begin{array}{c}\text { Antineoplastic }(0.833) \\
\text { Chemopreventive }(0.738) \\
\text { Proliferative diseases treatment }(0.643) \\
\text { Antimetastatic }(0.626)\end{array}$ & $\begin{array}{l}\text { Anti-hypercholesterolemic }(0.840) \\
\text { Hypolipemic }(0.646) \\
\text { Atherosclerosis treatment }(0.634)\end{array}$ & $\begin{array}{l}\text { Anti-inflammatory }(0.806) \\
\text { Anesthetic general }(0.800) \\
\text { Respiratory analeptic }(0.789) \\
\text { Antifungal }(0.616)\end{array}$ \\
\hline 3 & $\begin{array}{c}\text { Antineoplastic }(0.765) \\
\text { Dementia treatment }(0.512)\end{array}$ & $\begin{array}{c}\text { Anti-hypercholesterolemic }(0.655) \\
\text { Hypolipemic }(0.530)\end{array}$ & $\begin{array}{l}\text { Immunosuppressant }(0.735) \\
\text { Anti-inflammatory }(0.667)\end{array}$ \\
\hline 4 & $\begin{array}{l}\text { Antineoplastic }(0.798) \\
\text { Apoptosis agonist }(0.751) \\
\text { Antimetastatic }(0.524)\end{array}$ & $\begin{array}{c}\text { Hypolipemic }(0.732) \\
\text { Cholesterol synthesis inhibitor (0.545) } \\
\text { Antileukemic }(0.539)\end{array}$ & $\begin{array}{c}\text { Immunosuppressant }(0.759) \\
\text { Antifungal }(0.748) \\
\text { Antibacterial }(0.642) \\
\end{array}$ \\
\hline 6 & $\begin{array}{c}\text { Chemopreventive }(0.839) \\
\text { Antineoplastic }(0.834) \\
\text { Proliferative diseases treatment }(0.716) \\
\text { Antimetastatic }(0.573)\end{array}$ & $\begin{array}{c}\text { Anti-hypercholesterolemic }(0.937) \\
\text { Hypolipemic }(0.809) \\
\text { Neuroprotector }(0.767) \\
\text { Atherosclerosis treatment }(0.592)\end{array}$ & $\begin{array}{l}\text { Respiratory analeptic }(0.896) \\
\text { Anesthetic general }(0.828) \\
\text { Anti-inflammatory }(0.778) \\
\text { Antifungal }(0.768)\end{array}$ \\
\hline 7 & $\begin{array}{c}\text { Antineoplastic }(0.861) \\
\text { Chemopreventive }(0.827) \\
\text { Proliferative diseases treatment }(0.779) \\
\text { Anticarcinogenic }(0.565) \\
\text { Antimetastatic }(0.560)\end{array}$ & $\begin{array}{l}\text { Anti-hypercholesterolemic }(0.883) \\
\text { Hypolipemic }(0.771) \\
\text { Atherosclerosis treatment }(0.675)\end{array}$ & $\begin{array}{c}\text { Respiratory analeptic }(0.868) \\
\text { Antifungal }(0.821) \\
\text { Anti-inflammatory }(0.755) \\
\text { Antibacterial }(0.603) \\
\text { Antimycobacterial }(0.569)\end{array}$ \\
\hline 10 & $\begin{array}{c}\text { Antineoplastic }(0.776) \\
\text { Apoptosis agonist }(0.601)\end{array}$ & & $\begin{array}{l}\text { Antiprotozoal (Plasmodium) (0.778) } \\
\text { Anti-inflammatory }(0.765)\end{array}$ \\
\hline 11 & $\begin{array}{c}\text { Antineoplastic }(0.807) \\
\text { Apoptosis agonist }(0.747) \\
\text { Proliferative diseases treatment }(0.596)\end{array}$ & Anti-hypercholesterolemic $(0.630)$ & $\begin{array}{l}\text { Anti-inflammatory }(0.930) \\
\text { Anti-asthmatic }(0.627)\end{array}$ \\
\hline 12 & Antineoplastic (0.838) & & Anti-inflammatory (0.792) \\
\hline 13 & $\begin{array}{l}\text { Apoptosis agonist }(0.790) \\
\text { Antineoplastic }(0.781)\end{array}$ & & $\begin{array}{c}\text { Respiratory analeptic }(0.772) \\
\text { Immunosuppressant }(0.744) \\
\text { Antifungal }(0.708)\end{array}$ \\
\hline 14 & $\begin{array}{c}\text { Antineoplastic }(0.824) \\
\text { Proliferative diseases treatment }(0.610)\end{array}$ & $\begin{array}{l}\text { Anti-hypercholesterolemic }(0.909) \\
\text { Lipid metabolism regulator }(0.672)\end{array}$ & $\begin{array}{l}\text { Respiratory analeptic }(0.907) \\
\text { Anti-inflammatory }(0.673)\end{array}$ \\
\hline 15 & $\begin{array}{c}\text { Antineoplastic }(0.865) \\
\text { Proliferative diseases treatment }(0.812) \\
\text { Apoptosis agonist }(0.767) \\
\text { Anticarcinogenic }(0.730)\end{array}$ & $\begin{array}{l}\text { Anti-hypercholesterolemic }(0.930) \\
\text { Hypolipemic }(0.827) \\
\text { Atherosclerosis treatment }(0.646)\end{array}$ & $\begin{array}{c}\text { Respiratory analeptic }(0.970) \\
\text { Anti-inflammatory }(0.904) \\
\text { Anesthetic general }(0.850) \\
\text { Antibacterial }(0.586)\end{array}$ \\
\hline 16 & $\begin{array}{c}\text { Antineoplastic }(0.838) \\
\text { Proliferative diseases treatment (0.729) } \\
\text { Antimetastatic }(0.651)\end{array}$ & $\begin{array}{c}\text { Anti-hypercholesterolemic (0.909) } \\
\text { Hypolipemic }(0.786)\end{array}$ & $\begin{array}{c}\text { Respiratory analeptic }(0.937) \\
\text { Antifungal }(0.789) \\
\text { Cardiotonic }(0.626)\end{array}$ \\
\hline 17 & Antineoplastic (0.730) & Anti-hypercholesterolemic (0.679) & Immunosuppressant (0.722) \\
\hline 18 & $\begin{array}{c}\text { Antineoplastic }(0.925) \\
\text { Proliferative diseases treatment }(0.787) \\
\text { Prostate cancer treatment }(0.670)\end{array}$ & $\begin{array}{l}\text { Anti-hypercholesterolemic }(0.913) \\
\text { Hypolipemic }(0.626) \\
\text { Lipid metabolism regulator }(0.566)\end{array}$ & $\begin{array}{c}\text { Autoimmune disorders treatment }(0.908) \\
\text { Respiratory analeptic }(0.780) \\
\text { Antifungal }(0.525)\end{array}$ \\
\hline
\end{tabular}

*Pa is the probability of belonging to the class of "actives"; only activities with $\mathrm{Pa}>0.5$ are shown.

Steroids, $8 \alpha \mathrm{H}-3 \beta$,11-dihydroxy-5 $\alpha, 6 \alpha$-expoxy-24-methylene-9,11-secocholestan-9-one (4) and crassarosteroside A (5) were obtained from Sinularia granosa and S. crassa soft coral extracts [51,52], respectively. Steroids (3), 3 $\beta$-hydroxyergosta-5,24(28)-diene-7-one (6), ergosta-3 $\beta, 5 \alpha, 6 \beta$-triol (7) have been 
isolated from Sinularia conferta and S. nanolobata $[53,54]$ and compound (7) and $3 \beta, 7 \alpha$-dihydroxyergosta-5,24 (28)-diene (8) were detected in MeOH extract of the soft coral S. cruciata [55].

The reef soft coral S. brassica, which was cultured in an aquarium, afforded four steroids with methyl ester groups, sinubrasones A (9), B (10), D (11) and C (12). Compounds 9 and 10 were shown to exhibit significant cytotoxicity and compounds $\mathbf{1 1}$ and $\mathbf{1 2}$ were demonstrating attracting anti-inflammatory activities [56]. Steroids, 5,6ß-epoxy-gorgosterol (13) and leptosteroid (14) were isolated and structurally elucidated from the Vietnamese soft coral Sinularia leptoclados. Both compounds obtained showed significant cytotoxicity against HepG2 $\left(\mathrm{IC}_{50}=21.1 \mu \mathrm{M}\right)$ and colon adenocarcinoma $\left(\mathrm{IC}_{50}=28.6 \mu \mathrm{M}\right)$ cell lines [54].

The cytotoxic steroid, crassarosterol A (15), which was originally found in the soft coral S. crassa [57] was later found in S. arborea [58] and S. flexibilis [59]. Analysis of its biological activity has shown that it exhibits cytotoxicity toward K562 and MOLT-4 leukemia cancer cells. Originally a steroid, 24-methyl-5 $\beta$-cholest-24(28)-en- $1 \alpha, 3 \beta, 5 \beta$-triol-6-one, which was named gibberoketosterol (16) was identified from the lipophilic extracts of a Taiwanese soft coral S. gibberosa by Ahmed and co-workers in 2003 [60] and showed that to exhibit moderate cytotoxicity against the growth of Hepa59T/VGH cancer cells and it was later discovered in S. numerosa [61]. A methanol extract of the soft coral S. microspiculata revealed two sterols, 7-oxo-gorgosterol (17) and 16 $\alpha$-hydroxy-sarcosterol (18) [62]. Soft coral from Southern Taiwan S. leptoclados yielded a secosterol (19) [63].

The soft coral S. flexibilis appears to be one of the few corals most studied and described in the scientific literature. The structures of the steroids (19-36) are shown in Figure 2 and the potential biological activities are shown in Table 2. Yu and co-workers have used this organism to study steroids. Thus, a series of steroids (20-25) was isolated from the methanolic extract and their structures were

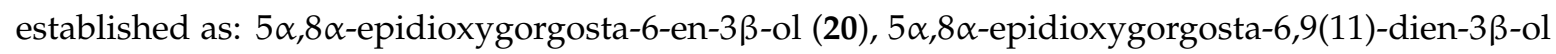

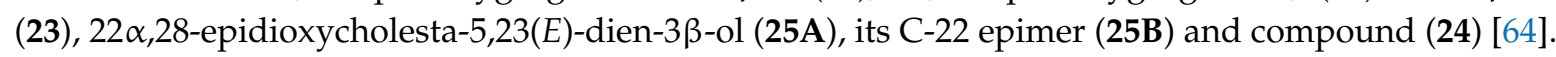
S. nanolobata yielded a sterol, sarcophytosterol (26), along with steroids (3) and (6) [65]. An unusual steroid, 3-(1', $2^{\prime}$-ethandiol)-cholest-3 $\beta, 5 \alpha, 6 \alpha, 11 \alpha$-tetraol (27) and secosteroid (28) were isolated from the South China sea gorgonian S. suberosa [66]. Two secosteroids (29) and (30) has been determined in Australian soft coral Sinularia sp. [67,68]. C18-Oxygenated steroidal ketal (31) was found in a Pacific soft coral extract of the genus Sinularia [69]. The minor sterol, ergosta-5,24(28),25-trien-3 $\beta$-ol (32), was isolated from the soft coral extract of the genus Sinularia, its structure was determined and it was synthesized [70] and soft coral S. dissecta yielded polyhydroxylated sterol (33) [71,72] and a similar sterol (34) detected in the S. numerosa [73].

Kobayashi and co-workers conducted studies of the soft coral S. mayi, S. gibberosa, S. dissecta and Sinularia sp. collected off the coast of Japan and identified a series of steroids (35-42) but the biological activity of the isolated steroids was not determined [74-78]. The structures of the steroids (37-58) are shown in Figure 3 and the potential biological activities are shown in Table 3. A $11 \alpha, 12 \alpha$-epoxy-steroid (43) was identified in S. dissecta [79], a $3 \beta, 6 \alpha, 9 \beta, 19 \alpha$-tetraol-steroid (44) was derived from S. inexplicita [80].

Octocoral S. leptoclados is a source of bioactive 9,11-secosteroids and steroid 3ß,11-dihydroxy-9,11secogorgost-5-en-9-one (45) showed the highest the $\gamma$-peroxisome proliferator-activated receptor (PPAR $\gamma$ ) activity with an $\mathrm{IC}_{50}$ value of $8.3 \mu \mathrm{M}$ for inhibiting human breast adenocarcinoma cell (MCF-7) growth. In addition, this steroid modulated the expression of various PPAR $\gamma$-regulated downstream biomarkers including cyclin D1, cyclin-dependent kinase, B-cell lymphoma 2 (Bcl-2), p38 and extracellular-signal-regulated kinase $[81,82]$ and two cytotoxic 5,8-epidioxy-steroid (46) and also (47) were found in Sinularia sp. [83,84]. Similar steroidal glycosides (48-51) were isolated from water-methanol solutions of the soft coral S. crispa [85], Sinularia sp. [86] and S. gibberosa [87].

Two cytotoxic secosteroids, $22 \alpha$-Acetoxy-24-methylene-3 $\beta, 6 \alpha, 11$-trihydroxy-9,11-seco-cholest-7-en -9-one (52) and 11-acetoxy-24-methylene-1 $\beta, 3 \beta, 6 \alpha$-trihydroxy-9,11-seco-cholest-7-en-9-one (53) have been isolated from the soft coral S. nanolobata [61]. 
Two unusual steroidal derivatives named erectsterates A (54) and B (55), a pair of epimers at C-10, were isolated from the South China Sea soft coral S. erecta. Both compounds are rare steroids with a high degradation in ring $B$ and an ester linkage between $A$ and $C / D$ rings, like the known compounds chaxines B and D from an edible mushroom Agrocybe chaxingu [88]. Compound (55) showed cytotoxic activity against A549 (human adenocarcinoma), HT-29 (human colorectal adenocarcinoma), SNU-398 (hepatocellular carcinoma) and Capan-1 (human pancreatic ductal adenocarcinoma) cancer cell lines [89].

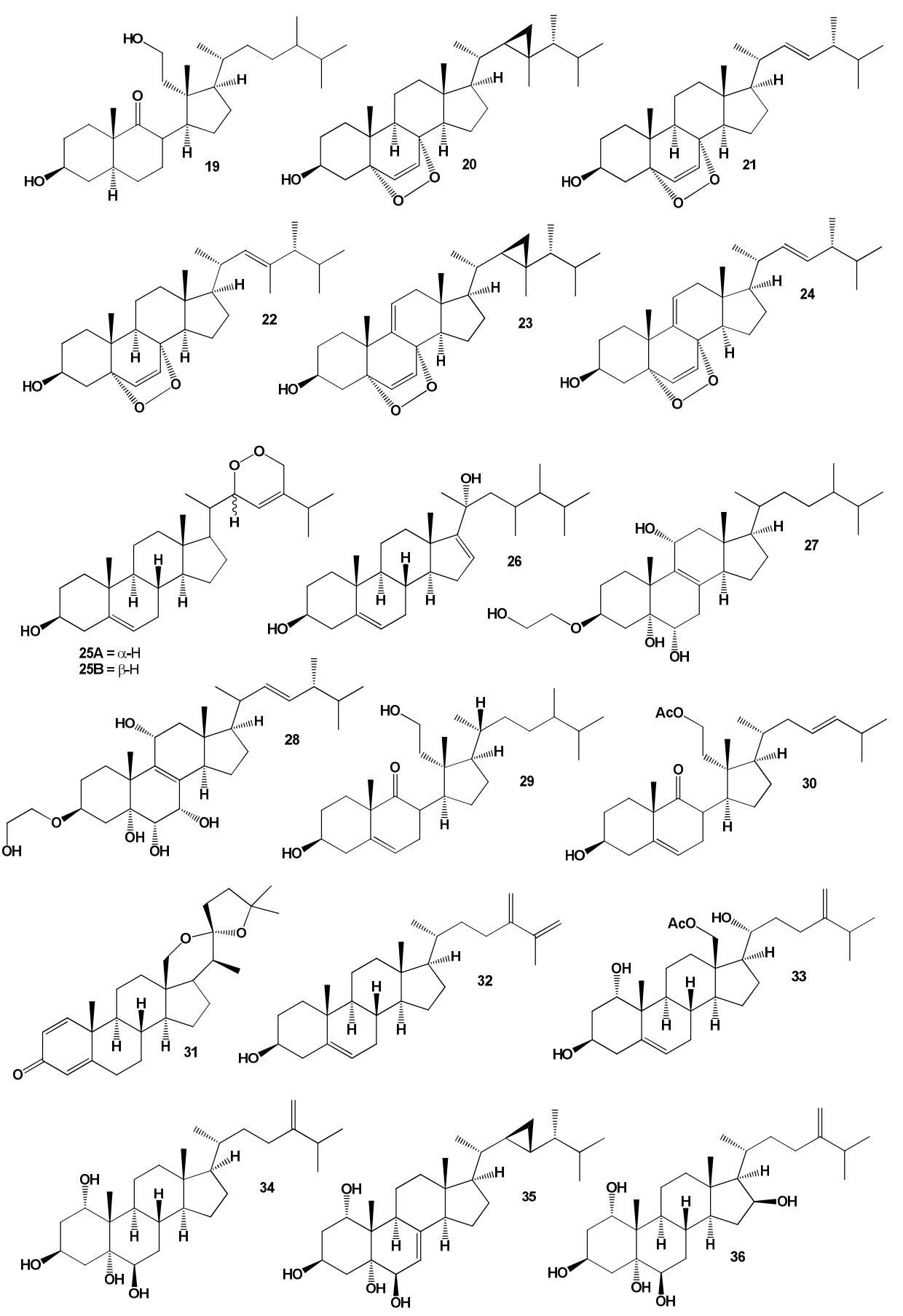

Figure 2. Bioactive steroids derived from the genus Sinularia. 
Table 2. Probable biological activities of steroids derived from the genus Sinularia estimated by PASS.

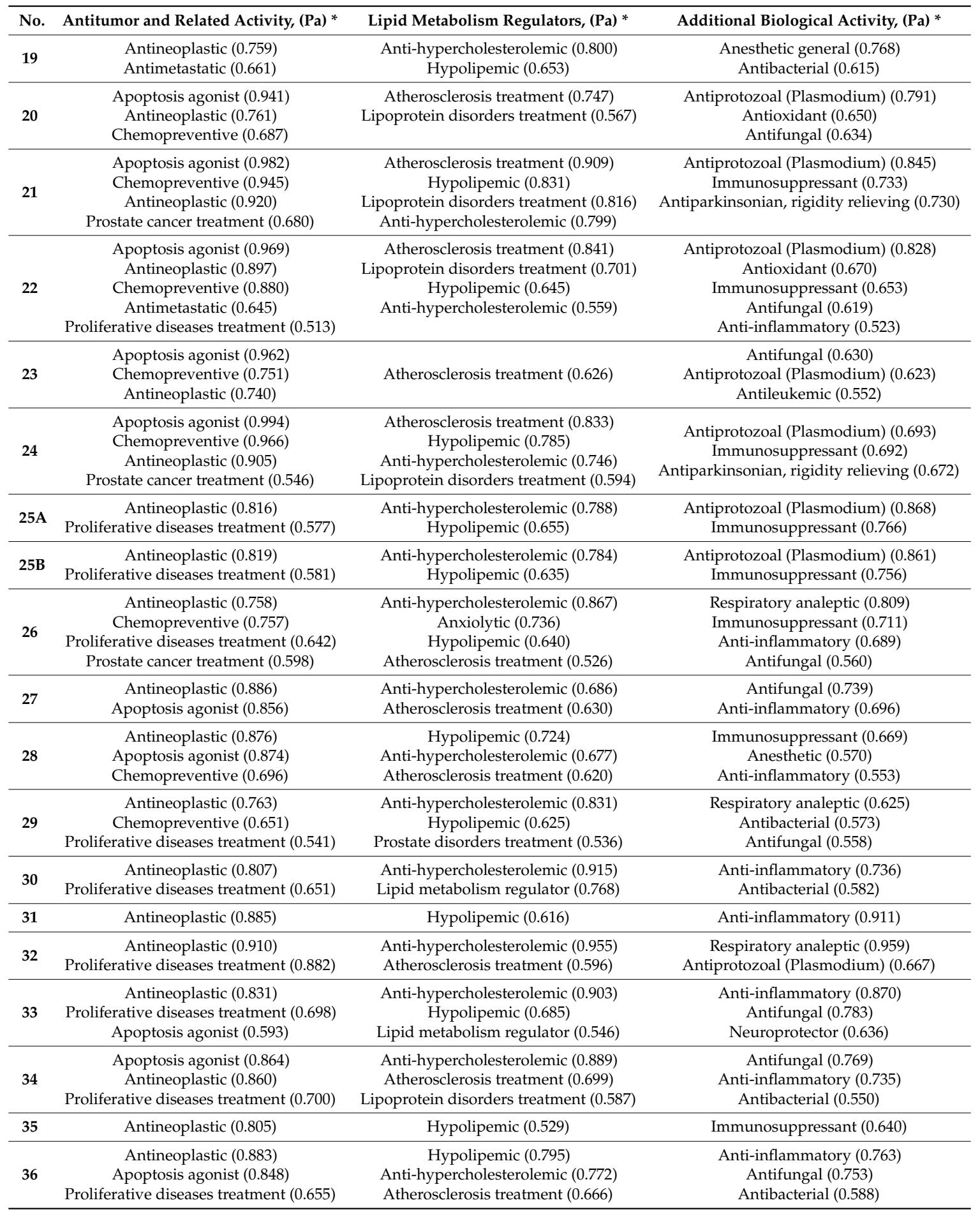

${ }^{*}$ Only activities with $\mathrm{Pa}>0.5$ are shown. 


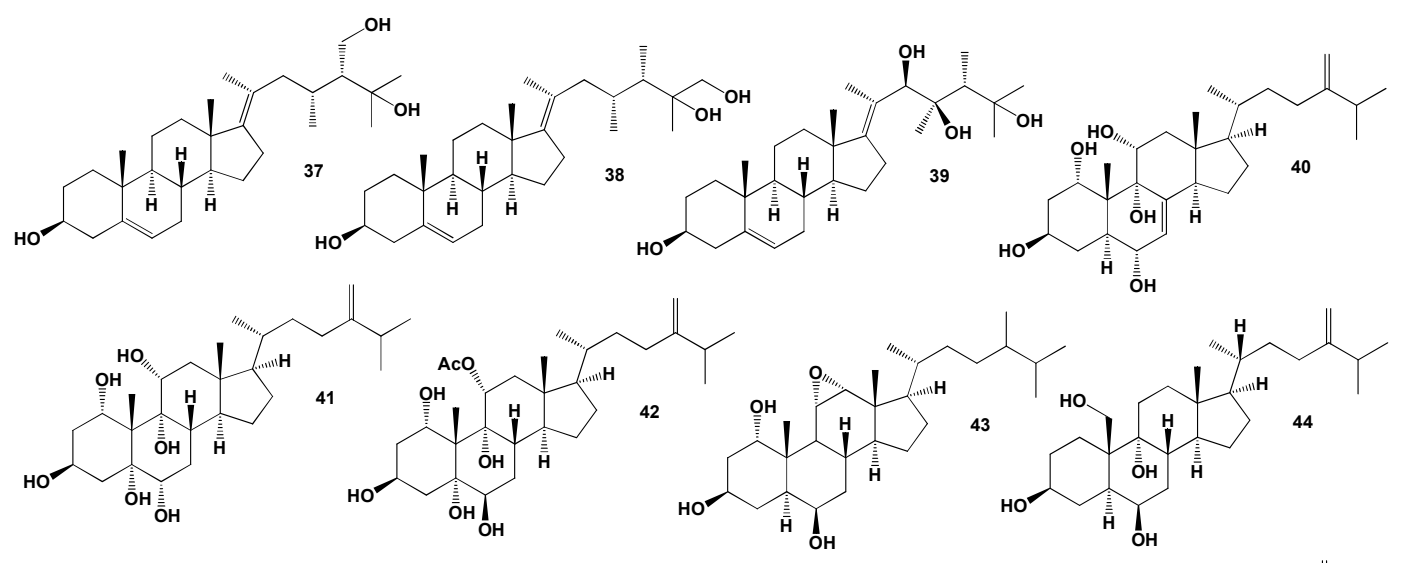

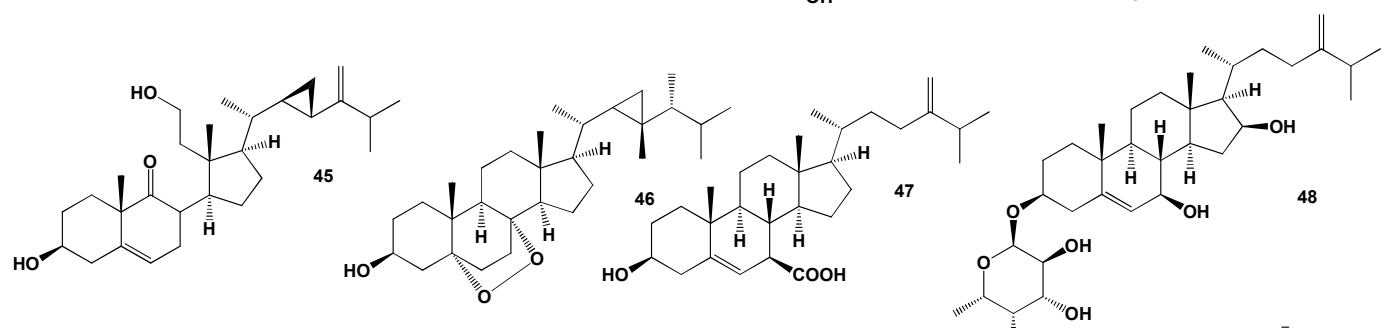

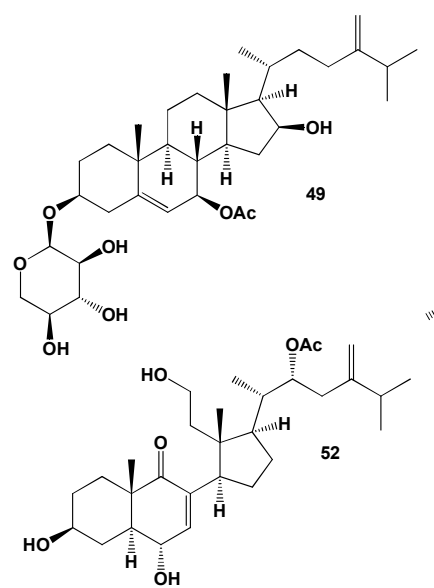

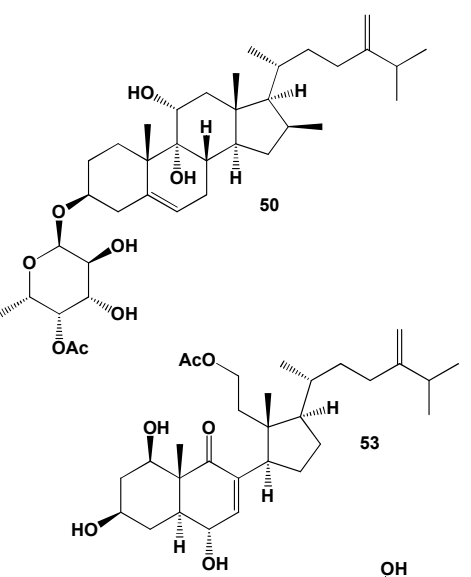

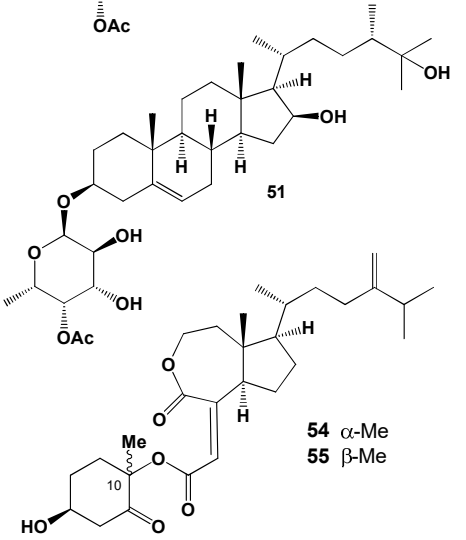<smiles>CC(C)C(C)C(C)CC1OC(=O)C23CCC4[C@H](CC=C5CC(O)CCC54C)[C@@H]2CC=C1C3</smiles>

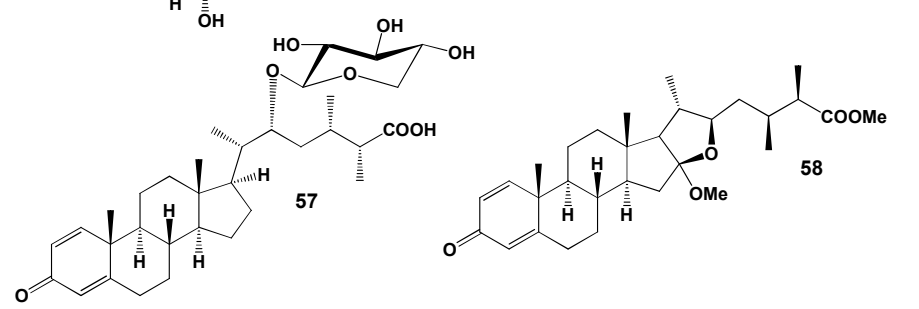

Figure 3. Bioactive steroids derived from the genus Sinularia.

Table 3. Biological activities of steroids derived from the genus Sinularia estimated by PASS.

\begin{tabular}{|c|c|c|c|}
\hline No. & Antitumor and Related Activity, $(\mathrm{Pa}) *$ & Lipid Metabolism Regulators, $(\mathrm{Pa})$ * & Additional Biological Activity, $(\mathrm{Pa})$ * \\
\hline \multirow{2}{*}{37} & Antineoplastic $(0.915)$ & Anti-hypercholesterolemic (0.910) & Autoimmune disorders treatment $(0.889)$ \\
\hline & Proliferative diseases treatment $(0.722)$ & Lipid metabolism regulator $(0.675)$ & Neuroprotector $(0.700)$ \\
\hline \multirow[t]{2}{*}{38} & Proliferative diseases treatment $(0.673)$ & Atherosclerosis treatment $(0.695)$ & Anti-inflammatory $(0.717)$ \\
\hline & & Lipid metabolism regulator $(0.575)$ & Neuroprotector (0.700) \\
\hline 39 & Antineoplastic (0.903) & Anti-hypercholesterolemic (0.947) & Autoimmune disorders treatment (0.829) \\
\hline \multirow{3}{*}{40} & Apoptosis agonist (0.823) & Hypolipemic $(0.901)$ & Antifungal (0.737) \\
\hline & Antineoplastic $(0.815)$ & Anti-hypercholesterolemic (0.754) & Anti-inflammatory (0.705) \\
\hline & Proliferative diseases treatment $(0.610)$ & Atherosclerosis treatment $(0.582)$ & Antibacterial (0.597) \\
\hline
\end{tabular}


Table 3. Cont.

\begin{tabular}{|c|c|c|c|}
\hline No. & Antitumor and Related Activity, $(\mathrm{Pa})$ * & Lipid Metabolism Regulators, $(\mathrm{Pa})$ * & Additional Biological Activity, (Pa) * \\
\hline 41 & $\begin{array}{l}\text { Apoptosis agonist }(0.845) \\
\quad \text { Antineoplastic }(0.823) \\
\text { Proliferative diseases treatment }(0.678)\end{array}$ & $\begin{array}{l}\text { Hypolipemic }(0.790) \\
\text { Anti-hypercholesterolemic }(0.687) \\
\text { Atherosclerosis treatment }(0.659)\end{array}$ & $\begin{array}{c}\text { Anti-fungal }(0.761) \\
\text { Anti-inflammatory }(0.735)\end{array}$ \\
\hline 42 & $\begin{array}{l}\text { Apoptosis agonist }(0.851) \\
\text { Antineoplastic }(0.824) \\
\text { Proliferative diseases treatment }(0.739)\end{array}$ & $\begin{array}{l}\text { Hypolipemic }(0.769) \\
\text { Anti-hypercholesterolemic }(0.733) \\
\text { Atherosclerosis treatment }(0.631)\end{array}$ & $\begin{array}{c}\text { Antifungal }(0.783) \\
\text { Anti-inflammatory }(0.775) \\
\text { Antibacterial }(0.543)\end{array}$ \\
\hline 44 & $\begin{array}{c}\text { Antineoplastic }(0.841) \\
\text { Apoptosis agonist }(0.770) \\
\text { Proliferative diseases treatment }(0.760)\end{array}$ & $\begin{array}{l}\text { Anti-hypercholesterolemic }(0.900) \\
\quad \text { Hypolipemic }(0.843) \\
\text { Atherosclerosis treatment }(0.620)\end{array}$ & $\begin{array}{c}\text { Cardiotonic }(0.872) \\
\text { Anesthetic general }(0.847) \\
\text { Antifungal }(0.787)\end{array}$ \\
\hline 45 & $\begin{array}{c}\text { Antineoplastic }(0.838) \\
\text { Proliferative diseases treatment (0.729) } \\
\text { Antimetastatic }(0.551)\end{array}$ & $\begin{array}{l}\text { Anti-hypercholesterolemic }(0.909) \\
\text { Hypolipemic }(0.786)\end{array}$ & $\begin{array}{l}\text { Antifungal }(0.789) \\
\text { Anti-inflammatory }(0.674) \\
\text { Cardiotonic }(0.626)\end{array}$ \\
\hline 47 & $\begin{array}{l}\text { Antineoplastic }(0.790) \\
\text { Proliferative diseases treatment }(0.652)\end{array}$ & $\begin{array}{l}\text { Anti-hypercholesterolemic }(0.963) \\
\text { Atherosclerosis treatment }(0.742)\end{array}$ & $\begin{array}{c}\text { Antifungal }(0.798) \\
\text { Anti-inflammatory }(0.722)\end{array}$ \\
\hline 48 & $\begin{array}{c}\text { Proliferative diseases treatment }(0.953) \\
\text { Anticarcinogenic }(0.922) \\
\text { Antineoplastic }(0.911)\end{array}$ & $\begin{array}{l}\text { Anti-hypercholesterolemic }(0.962) \\
\text { Hypolipemic }(0.825) \\
\text { Atherosclerosis treatment }(0.588)\end{array}$ & $\begin{array}{l}\text { Neuroprotector }(0.976) \\
\text { Antimycobacterial }(0.929) \\
\text { Antifungal }(0.885)\end{array}$ \\
\hline 49 & $\begin{array}{c}\text { Chemopreventive }(0.960) \\
\text { Proliferative diseases treatment }(0.945) \\
\text { Anticarcinogenic }(0.881) \\
\text { Antineoplastic }(0.877)\end{array}$ & $\begin{array}{c}\text { Anti-hypercholesterolemic }(0.964) \\
\text { Hypolipemic }(0.797)\end{array}$ & $\begin{array}{l}\text { Antimycobacterial }(0.936) \\
\text { Antifungal }(0.847) \\
\text { Anti-inflammatory }(0.823) \\
\text { Neuroprotector }(0.757)\end{array}$ \\
\hline 50 & $\begin{array}{c}\text { Chemopreventive }(0.953) \\
\text { Proliferative diseases treatment }(0.944) \\
\text { Antineoplastic }(0.858)\end{array}$ & $\begin{array}{l}\text { Anti-hypercholesterolemic }(0.943) \\
\text { Hypolipemic }(0.846) \\
\text { Atherosclerosis treatment }(0.510)\end{array}$ & $\begin{array}{c}\text { Respiratory analeptic }(0.957) \\
\text { Neuroprotector }(0.892) \\
\text { Antifungal }(0.873)\end{array}$ \\
\hline 53 & $\begin{array}{l}\text { Antineoplastic }(0.790) \\
\text { Proliferative diseases treatment }(0.667)\end{array}$ & $\begin{array}{l}\text { Anti-hypercholesterolemic (0.729) } \\
\text { Cholesterol synthesis inhibitor }(0.664)\end{array}$ & $\begin{array}{l}\text { Anti-osteoporotic }(0.965) \\
\text { Anti-eczematic }(0.910)\end{array}$ \\
\hline 54 & $\begin{array}{c}\text { Antineoplastic }(0.790) \\
\text { Proliferative diseases treatment }(0.767)\end{array}$ & $\begin{array}{l}\text { Anti-hypercholesterolemic }(0.729) \\
\text { Cholesterol synthesis inhibitor }(0.614)\end{array}$ & $\begin{array}{l}\text { Anti-osteoporotic }(0.965) \\
\text { Anti-eczematic }(0.910)\end{array}$ \\
\hline 55 & $\begin{array}{l}\text { Antineoplastic }(0.948) \\
\text { Alzheimer's disease treatment }(0.851) \\
\text { Apoptosis agonist }(0.696)\end{array}$ & $\begin{array}{c}\text { Anti-hypercholesterolemic }(0.821) \\
\text { Neurodegenerative diseases treatment } \\
(0.815) \\
\text { Cholesterol synthesis inhibitor }(0.741)\end{array}$ & $\begin{array}{c}\text { Immunomodulator (HIV) }(0.893) \\
\text { Antioxidant }(0.821)\end{array}$ \\
\hline 56 & $\begin{array}{c}\text { Chemopreventive }(0.918) \\
\text { Proliferative diseases treatment }(0.874) \\
\text { Antineoplastic }(0.845)\end{array}$ & $\begin{array}{c}\text { Anti-hypercholesterolemic }(0.914) \\
\text { Acute neurologic disorders treatment } \\
(0.632) \\
\text { Atherosclerosis treatment }(0.612)\end{array}$ & $\begin{array}{l}\text { Anti-inflammatory }(0.870) \\
\text { Immunosuppressant }(0.818)\end{array}$ \\
\hline 57 & $\begin{array}{c}\text { Antineoplastic }(0.784) \\
\text { Apoptosis agonist }(0.654) \\
\text { Prostate disorders treatment }(0.625)\end{array}$ & & $\begin{array}{l}\text { Antiprotozoal (Plasmodium) }(0.766) \\
\text { Anti-inflammatory }(0.752) \\
\text { Immunosuppressant }(0.705)\end{array}$ \\
\hline 58 & $\begin{array}{c}\text { Antineoplastic }(0.795) \\
\text { Apoptosis agonist }(0.711) \\
\text { Prostate disorders treatment }(0.633)\end{array}$ & & $\begin{array}{l}\text { Antiprotozoal (Plasmodium) (0.754) } \\
\text { Anti-inflammatory }(0.732) \\
\text { Immunosuppressant }(0.712)\end{array}$ \\
\hline
\end{tabular}

* Only activities with $\mathrm{Pa}>0.5$ are shown.

A steroid named dissectolide (56) was purified from the methanol extract of soft coral S. dissecta [90]. The isolated steroid inhibits the growth of Balanus amphitrite larvae [91]. The ethyl acetate extract of a reef soft coral S. brassica, which was cultured in a tank, afforded two steroids, sinubrasones A (57) and B (58). Both highlighted products to exhibit significant cytotoxicity [92]. 


\section{Steroids Derived from the other Coral's Genera}

The 17 $\beta, 20 \beta$-epoxy-23,24-dimethylcholest-5-ene-3 $\beta$, 22-diacetate (59) and three unusual steroids (60-62) including secosteroid (62) were isolated from the Indian Ocean soft coral Sarcophyton crassocaula [93]. Cytotoxic steroids (63-67) were obtained from the acetone and $\mathrm{MeOH}$ extract of the soft coral Nephthea erecta [94]. A steroid with a spiro-ring A, B system named chabrolosteroid C (66) and chabrolosteroid A (68) were isolated from an organic extract of a Taiwanese soft coral Nephthea chabrolii [95]. The soft coral Umbellulifera petasites produces the steroid (68) and petasiterone B (69), as well as $5 \alpha$-pregna-20-en-3-one

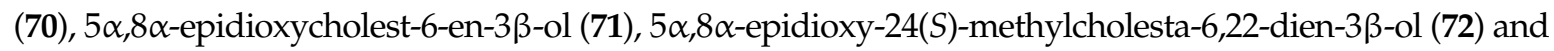

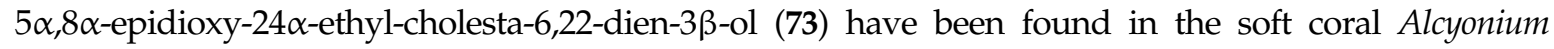
gracillimum [96-98]. The structures of the steroids (59-84) are shown in Figure 4 and the potential biological activities are shown in Table 4.
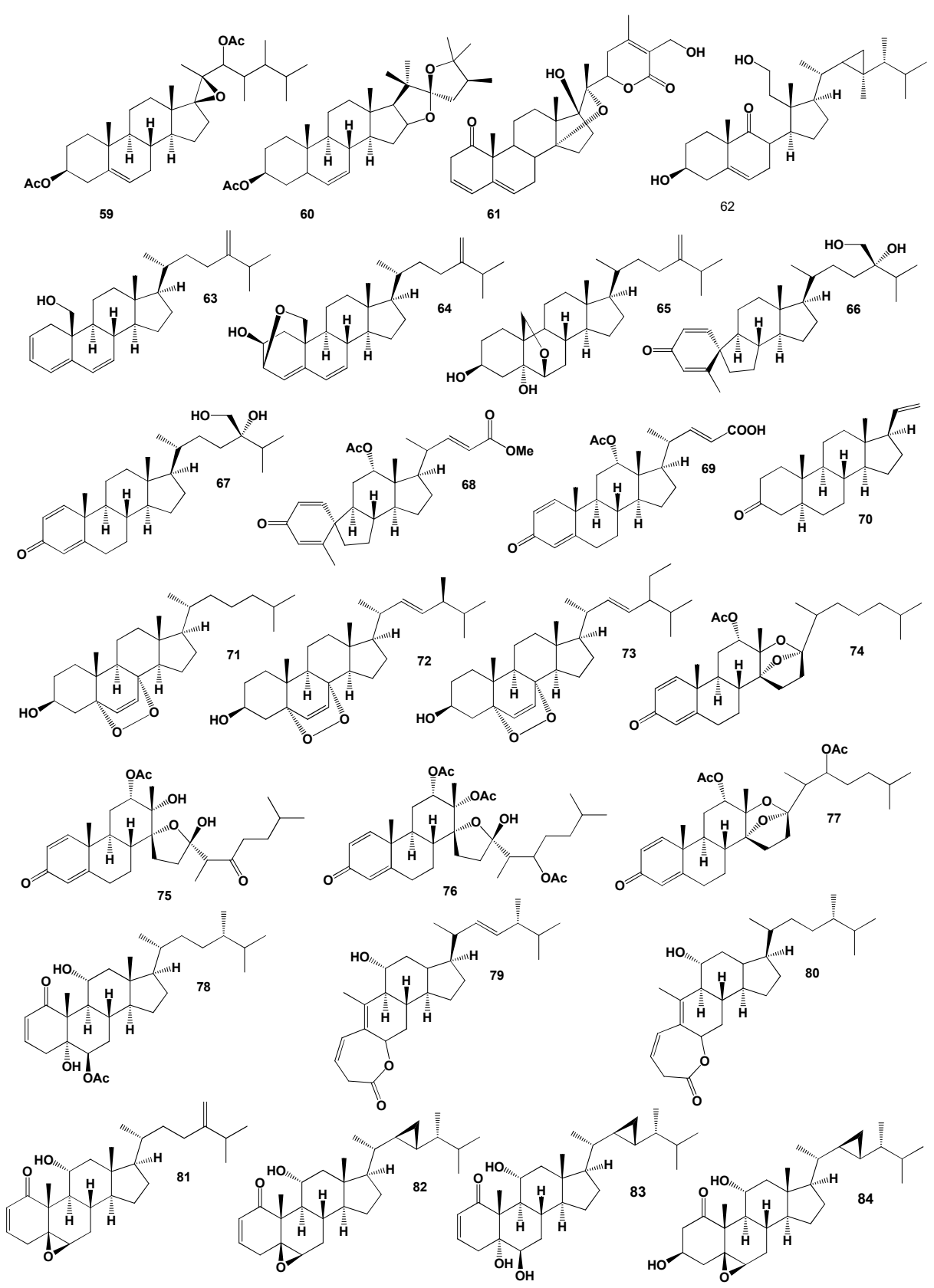

Figure 4. Bioactive steroids derived from soft corals. 
Unique highly oxygenated 13,17-secosteroids with split D ring were obtained from extracts of a Japanese octocoral of the genus Dendronephthya collected off the Izu Peninsula and named isogosterones A-D (74-77). The resulting steroids have inhibited the settlement of the B. amphitrite cyprid larvae [99]. Steroid, named 6-epi-yonarasterol B (78) was found in the Formosan gorgonian coral Echinomuricea sp. (family Plexauridae) [100].

Lactonic steroid derivatives with an unprecedented 1,10-secoergostane skeleton, stoloniolide I and II (79 and 80) and stoloniferones A (81), D (82), J (83), L (84) and O (85), which showed cytotoxic activity were found in the Okinawan soft coral Clavularia viridis [101-104].

Table 4. Probable biological activities of steroids derived from soft coral species estimated by PASS.

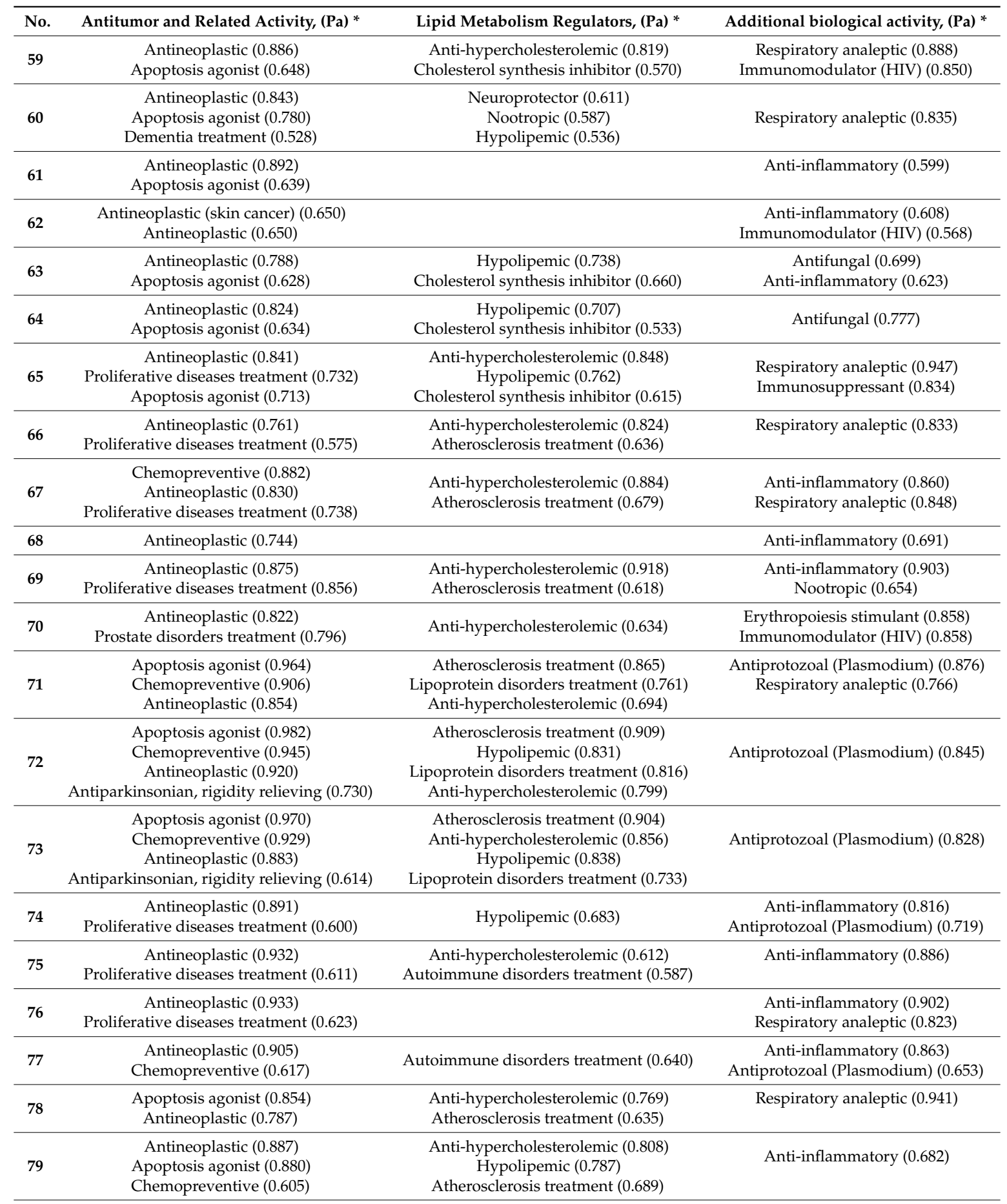


Table 4. Cont.

\begin{tabular}{|c|c|c|c|}
\hline No. & Antitumor and Related Activity, $(\mathrm{Pa})$ * & Lipid Metabolism Regulators, (Pa) * & Additional biological activity, $(\mathrm{Pa})$ * \\
\hline \multirow{2}{*}{80} & Antineoplastic (0.799) & Atherosclerosis treatment $(0.611)$ & Antifungal (0.751) \\
\hline & Apoptosis agonist (0.701) & Cholesterol synthesis inhibitor (0.589) & Antithrombotic (0.558) \\
\hline \multirow{2}{*}{81} & Antineoplastic (0.877) & Anti-hypercholesterolemic (0.638) & Respiratory analeptic (0.945) \\
\hline & Apoptosis agonist (0.838) & Cholesterol synthesis inhibitor (0.628) & \\
\hline 82 & Antineoplastic (0.857) & & Antifungal (0.786) \\
\hline \multirow{2}{*}{83} & Antineoplastic $(0.753)$ & & Anti-inflammatory (0.733) \\
\hline & Alzheimer's disease treatment $(0.674)$ & & Immunosuppressant $(0.723)$ \\
\hline \multirow{2}{*}{84} & Antineoplastic (0.852) & & Respiratory analeptic $(0.871)$ \\
\hline & Apoptosis agonist $(0.760)$ & & Immunosuppressant (0.781) \\
\hline
\end{tabular}

Polyhydroxygenated steroids, hipposterone M (86), hipposterol G (87) and hippuristeroketal A (88) that demonstrated cytotoxicity against the anti-HCMV (human cytomegalovirus) were obtained from extracts of Taiwanese octocoral Isis hippuris collected at Orchid Island [105]. The structures of the steroids (85-111) are shown in Figure 5 and the potential biological activities are shown in Tables 5 and 6. Steroid, named erectasteroid H (89), showed cytotoxic activity against P-388 (leukemia) and HT-29 [106] and spirosteroid (90) have been isolated from the Formosan soft coral N. erecta $[107,108]$.

The coral Klyxum flaccidum produced the secosteroid, klyflaccisteroid K (91), which showed significant anti-inflammatory activity in suppressing superoxide anion generation and elastase release, with $\mathrm{IC}_{50}$ values of 5.8 and $1.5 \mu \mathrm{M}$, respectively [109] and the coral Pinnigorgia sp. produces bioactive 9,11-secosteroids, pinnisteroids A and C (92 and 93) displayed remarkable inhibitory effects on the generation of superoxide anions and the release of elastase in human neutrophils, with $\mathrm{IC}_{50}$ values from 2.3 to $3.3 \mu \mathrm{M}$ [110]. The soft coral Lobophytum laevigatum contained unusual steroid, (22S,24S)-24-methyl-22, 25-epoxyfurost-5-ene-3 $\beta, 20 \beta$-diol (94) and demonstrated significantly upregulated PPAR transcriptional activity dose-dependently in Hep-G2 cells [111].

The 9,11-secosteroids, pinnigorgiols A (95), B (96) and E (97) with a rare carbon skeleton, a tricyclo[5,2,1]decane ring, were isolated from a gorgonian coral identified as Pinnigorgia sp. Isolated compounds displayed inhibitory effects on the generation of superoxide anions and the release of elastase by human neutrophils [112,113]. 16,22-Epoxy-20ß,23S-dihydroxycholest-1-ene-3-one (98) unusual cholestane derivatives, was isolated from the South China Sea gorgonian coral Subergorgia suberosa.

A series of cytotoxic steroids, verrucorosteroids A (99), B (100), D (101) and F (102), which demonstrated anticancer activity against eight human cancer cell lines as LNCaP (prostate cancer), HepG2, KB (epidermoid carcinoma), MCF-7, SK-Mel2 (melanoma), HL-60, LU-1 (lung cancer) and SW480 were isolated from the Vietnamese gorgonian Verrucella corona [114].

A series of steroids (103-111) was found and isolated for studying their biological activity from the genus Alcyonium. A steroid isolated from the Formosan soft coral Alcyonium sp. (Alcyoniidae) $3 \alpha, 7 \alpha, 12 \alpha$-triacetoxy-5 $\beta$-cholanic acid (103) [115] and steroids (104-107) were obtained from the crude extract of $A$. gracillimum which exhibited moderate cytotoxicity $\left(\mathrm{IC}_{50}\right.$ $22 \mu \mathrm{g} / \mathrm{mL}$ ) and antiviral activity ( $\mathrm{IC}_{50} 8 \mu \mathrm{g} / \mathrm{mL}$ ) against P388 and HSV-I (human $\alpha$-herpesvirus), respectively [116,117]. Steroid derivatives 3-methoxy-19-norpregna-1,3,5(10),20-tetraene (108), 3-(4-O-acetyl6-deoxy- $\beta$-galactopyranosyloxy)-19-norpregna-1,3,5(10),20-tetraene (109) were isolated from A. gracillimum, which was collected from the Gulf of Sagami, Japan [118] and cytotoxic

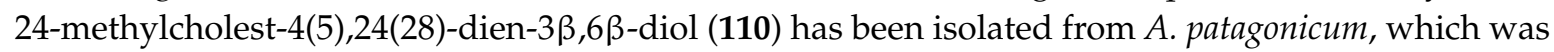
collected from the South China Sea [119]. The acetone extract of Alcyonium sp., which was collected from Taketomijima, Okinawa, yielded steroid, 3'-O-acetyl-pregnedioside-A (111) [120].

Another series of cytotoxic steroids called stereonsteroids A (112), B (113), D (114), F (115) and G (116, activity is shown in Table 7) were isolated from the methylene chloride extract of the Formosan soft coral Stereonephthya crystalliana. The extract of this coral showed significant cytotoxicity against A549, HT-29 and P-388 cancer cells in vitro [121]. Another two cytotoxic named sclerosteroids D (117) and E (118) were found in the soft coral Scleronephthya gracillimum [122]. Pregnane derivative 
4-hydroxymethyl-5 $\beta$-pregnan-3, 20-dione (119) has been isolated from the South China Sea gorgonian Subergorgia suberosa [123].

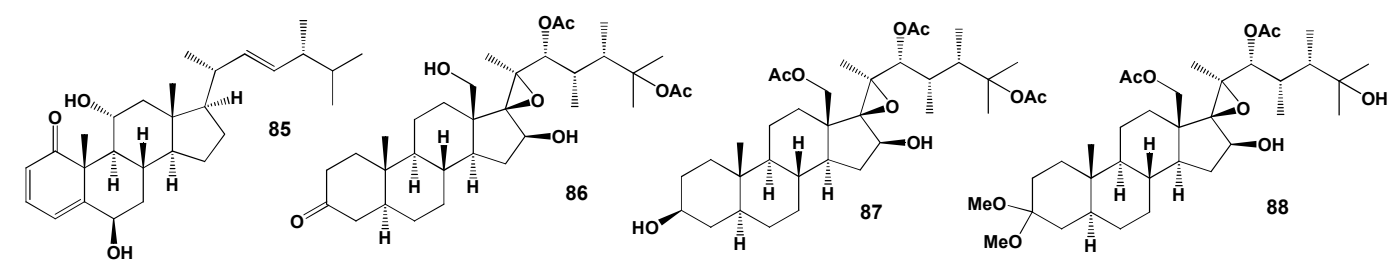

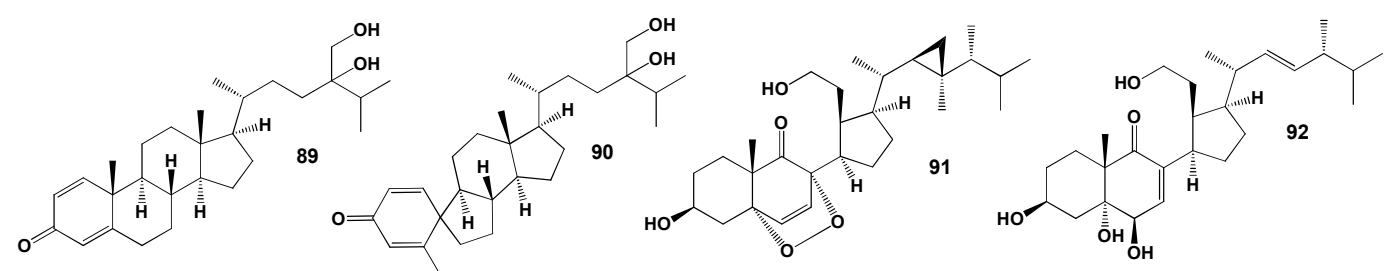

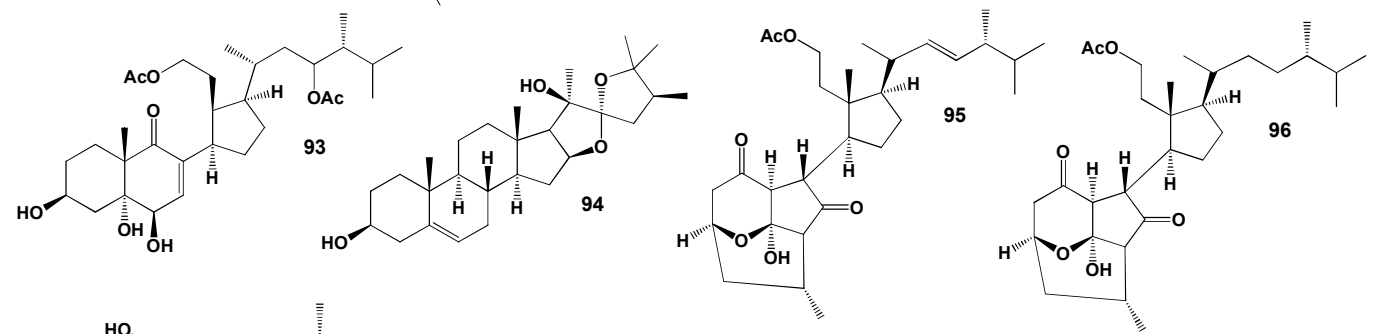

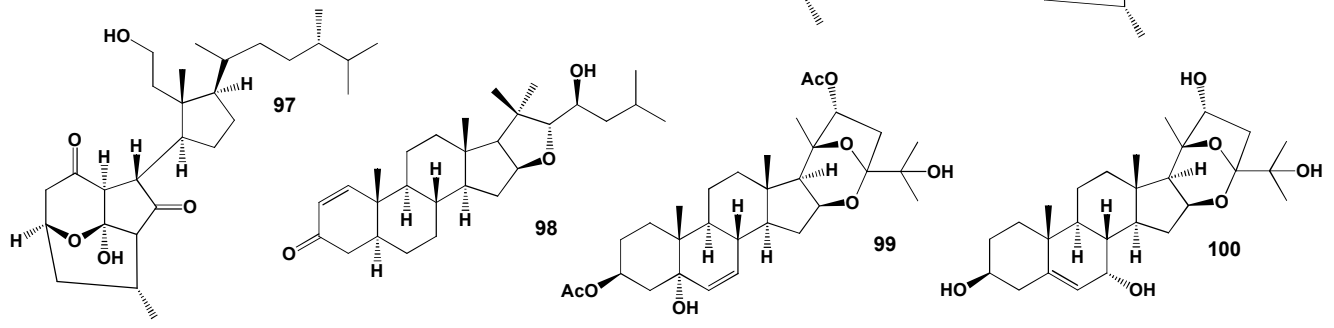

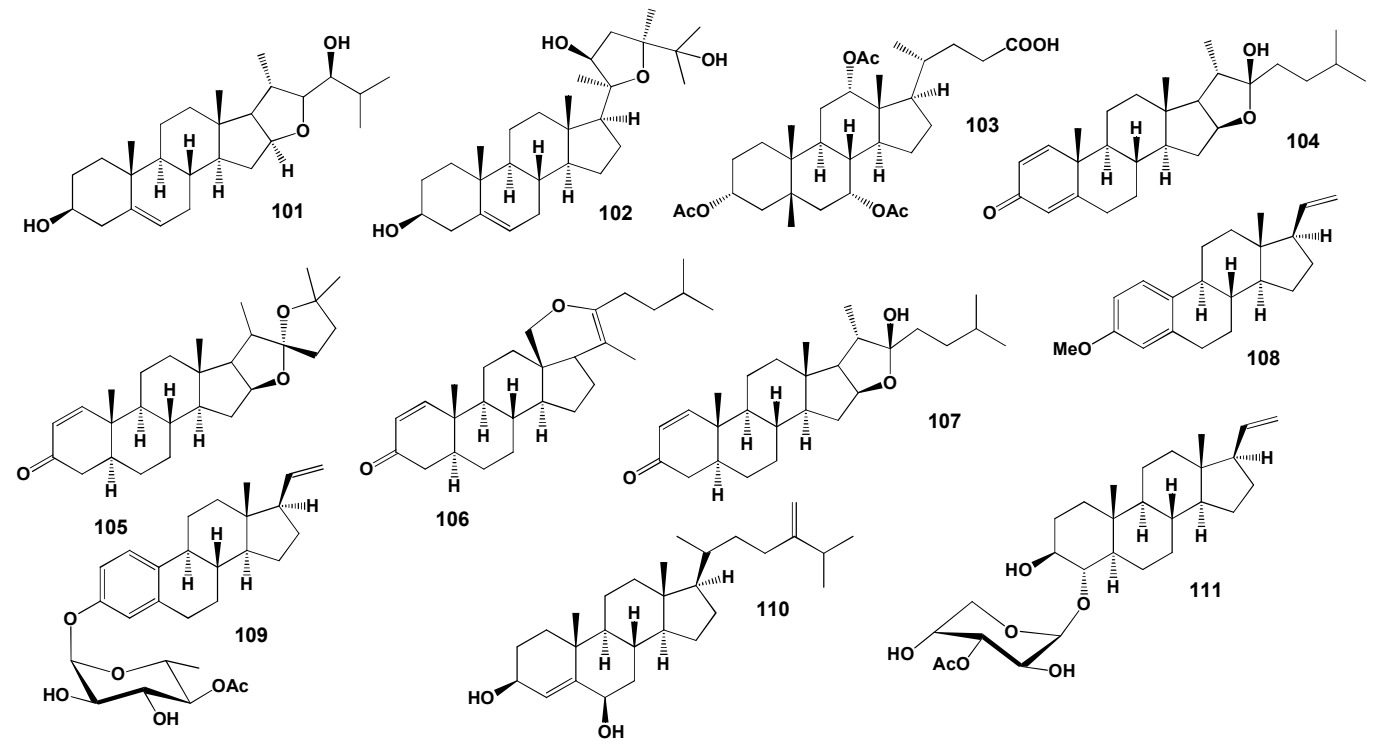

Figure 5. Bioactive steroids derived from soft corals. 
Table 5. Probable biological activities of steroids derived from soft coral species estimated by PASS.

\begin{tabular}{|c|c|c|c|}
\hline No. & Antitumor and Related Activity, $(\mathrm{Pa}) *$ & Lipid Metabolism Regulators, $(\mathrm{Pa})$ * & Additional Biological Activity, (Pa) * \\
\hline 85 & $\begin{array}{c}\text { Apoptosis agonist }(0.902) \\
\text { Antineoplastic }(0.878) \\
\text { Antiparkinsonian, rigidity relieving } \\
(0.507)\end{array}$ & $\begin{array}{l}\text { Anti-hypercholesterolemic }(0.894) \\
\text { Hypolipemic }(0.785) \\
\text { Atherosclerosis treatment }(0.650)\end{array}$ & $\begin{array}{l}\text { Anti-inflammatory }(0.829) \\
\text { Immunosuppressant }(0.799)\end{array}$ \\
\hline 86 & Antineoplastic (0.891) & $\begin{array}{l}\text { Anti-hypercholesterolemic }(0.841) \\
\text { Neuroprotector }(0.727)\end{array}$ & $\begin{array}{l}\text { Angiogenesis inhibitor }(0.910) \\
\text { Respiratory analeptic }(0.848)\end{array}$ \\
\hline 87 & Antineoplastic (0.864) & $\begin{array}{l}\text { Anti-hypercholesterolemic }(0.836) \\
\text { Neuroprotector }(0.806)\end{array}$ & $\begin{array}{c}\text { Anesthetic general (0.910) } \\
\text { Angiogenesis inhibitor (0.895) }\end{array}$ \\
\hline 88 & Antineoplastic (0.868) & $\begin{array}{c}\text { Anti-hypercholesterolemic }(0.623) \\
\text { Hypolipemic }(0.521)\end{array}$ & $\begin{array}{l}\text { Angiogenesis inhibitor }(0.765) \\
\text { Anti-asthmatic }(0.630)\end{array}$ \\
\hline 89 & $\begin{array}{l}\text { Chemopreventive }(0.882) \\
\text { Antineoplastic }(0.830) \\
\text { Apoptosis agonist }(0.668)\end{array}$ & $\begin{array}{l}\text { Anti-hypercholesterolemic }(0.884) \\
\text { Atherosclerosis treatment }(0.679) \\
\text { Hypolipemic }(0.623)\end{array}$ & $\begin{array}{l}\text { Anti-inflammatory }(0.860) \\
\text { Respiratory analeptic }(0.848) \\
\text { Anti-asthmatic }(0.542)\end{array}$ \\
\hline 90 & $\begin{array}{c}\text { Chemopreventive }(0.761) \\
\text { Antineoplastic }(0.761)\end{array}$ & $\begin{array}{l}\text { Anti-hypercholesterolemic }(0.824) \\
\text { Atherosclerosis treatment }(0.636)\end{array}$ & $\begin{array}{l}\text { Respiratory analeptic }(0.833) \\
\text { Immunosuppressant }(0.740)\end{array}$ \\
\hline 91 & $\begin{array}{l}\text { Apoptosis agonist }(0.767) \\
\text { Antineoplastic }(0.597)\end{array}$ & $\begin{array}{l}\text { Hypolipemic }(0.520) \\
\text { Atherosclerosis treatment }(0.500)\end{array}$ & $\begin{array}{c}\text { Antiprotozoal }(0.723) \\
\text { Immunosuppressant }(0.534)\end{array}$ \\
\hline 92 & $\begin{array}{c}\text { Antineoplastic }(0.871) \\
\text { Apoptosis agonist }(0.859)\end{array}$ & $\begin{array}{l}\text { Anti-hypercholesterolemic }(0.832) \\
\text { Atherosclerosis treatment }(0.687)\end{array}$ & $\begin{array}{c}\text { Nootropic }(0.744) \\
\text { Immunosuppressant }(0.733)\end{array}$ \\
\hline 93 & $\begin{array}{c}\text { Antineoplastic }(0.812) \\
\text { Apoptosis agonist }(0.737) \\
\text { Chemopreventive }(0.650)\end{array}$ & $\begin{array}{l}\text { Hypolipemic }(0.764) \\
\text { Anti-hypercholesterolemic }(0.686) \\
\text { Atherosclerosis treatment }(0.575)\end{array}$ & $\begin{array}{l}\text { Immunosuppressant }(0.652) \\
\text { Nootropic }(0.602)\end{array}$ \\
\hline 94 & $\begin{array}{c}\text { Antineoplastic }(0.935) \\
\text { Apoptosis agonist }(0.833)\end{array}$ & Anti-hypercholesterolemic (0.789) & $\begin{array}{c}\text { Immunomodulator (HIV) }(0.958) \\
\text { Respiratory analeptic }(0.923)\end{array}$ \\
\hline 95 & $\begin{array}{c}\text { Antineoplastic }(0.860) \\
\text { Apoptosis agonist }(0.844)\end{array}$ & $\begin{array}{c}\text { Hypolipemic }(0.748) \\
\text { Anti-hypercholesterolemic }(0.536)\end{array}$ & $\begin{array}{l}\text { Immunosuppressant }(0.773) \\
\text { Allergic conjunctivitis treatment }(0.608)\end{array}$ \\
\hline 96 & Antineoplastic (0.773) & Hypolipemic (0.595) & Anti-eczematic (0.864) \\
\hline 97 & Antineoplastic (0.776) & Hypolipemic (0.559) & Anti-eczematic (0.856) \\
\hline 98 & Antineoplastic (0.809) & Anti-hypercholesterolemic (0.672) & Respiratory analeptic (0.935) \\
\hline
\end{tabular}

* Only activities with $\mathrm{Pa}>0.5$ are shown.

Table 6. Probable biological activities of steroids derived from soft coral species estimated by PASS.

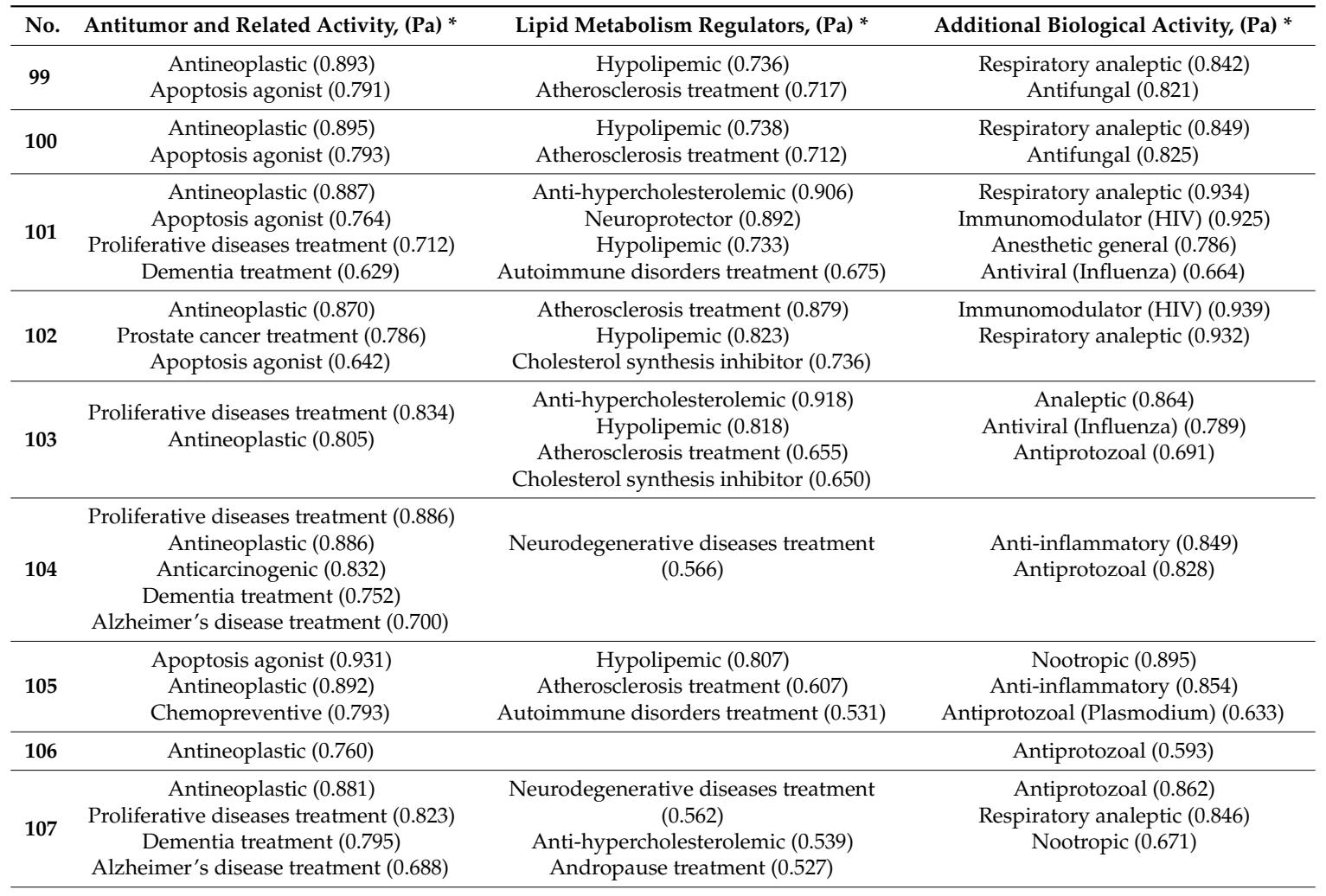


Table 6. Cont.

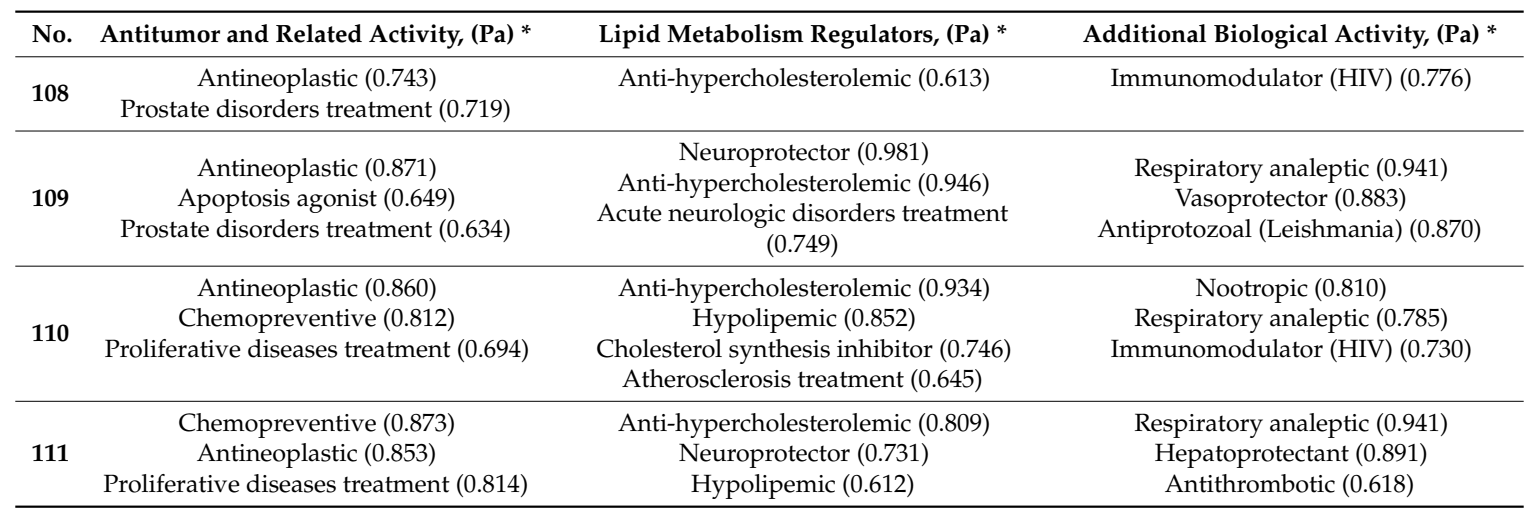

* Only activities with $\mathrm{Pa}>0.5$ are shown.

Table 7. Probable biological activities of steroids derived from soft coral species estimated by PASS.

\begin{tabular}{|c|c|c|c|}
\hline No. & Antitumor and Related Activity, $(\mathrm{Pa})$ * & Lipid Metabolism Regulators, $(\mathrm{Pa}) *$ & Additional Biological Activity, $(\mathrm{Pa})$ * \\
\hline 112 & $\begin{array}{c}\text { Antineoplastic }(0.864) \\
\text { Apoptosis agonist }(0.850) \\
\text { Prostate disorders treatment }(0.705) \\
\text { Dementia treatment }(0.629)\end{array}$ & $\begin{array}{c}\text { Neuroprotector }(0.786) \\
\text { Anti-hypercholesterolemic }(0.774) \\
\text { Cholesterol synthesis inhibitor }(0.524)\end{array}$ & $\begin{array}{l}\text { Immunomodulator (HIV) }(0.895) \\
\text { Erythropoiesis stimulant }(0.894) \\
\text { Anesthetic general }(0.865)\end{array}$ \\
\hline 113 & $\begin{array}{c}\text { Antineoplastic }(0.848) \\
\text { Apoptosis agonist }(0.826) \\
\text { Proliferative diseases treatment }(0.608)\end{array}$ & $\begin{array}{l}\text { Anti-hypercholesterolemic }(0.811) \\
\text { Lipid metabolism regulator }(0.673)\end{array}$ & $\begin{array}{c}\text { Respiratory analeptic }(0.911) \\
\text { Neuroprotector }(0.825) \\
\text { Immunomodulator }(\mathrm{HIV})(0.820)\end{array}$ \\
\hline 114 & $\begin{array}{c}\text { Proliferative diseases treatment }(0.923) \\
\text { Antineoplastic }(0.889) \\
\text { Chemopreventive }(0.853) \\
\text { Apoptosis agonist }(0.741) \\
\text { Dementia treatment }(0.673)\end{array}$ & $\begin{array}{c}\text { Neuroprotector }(0.950) \\
\text { Anti-hypercholesterolemic }(0.821) \\
\text { Spasmolytic }(0.684) \\
\text { Hypolipemic }(0.621)\end{array}$ & $\begin{array}{l}\text { Respiratory analeptic }(0.981) \\
\text { Anesthetic general }(0.918) \\
\text { Cardiotonic }(0.813) \\
\text { Antithrombotic }(0.567)\end{array}$ \\
\hline 115 & $\begin{array}{c}\text { Chemopreventive }(0.942) \\
\text { Antineoplastic }(0.901) \\
\text { Apoptosis agonist }(0.837) \\
\text { Proliferative diseases treatment }(0.788) \\
\text { Dementia treatment }(0.665)\end{array}$ & $\begin{array}{l}\text { Neuroprotector }(0.971) \\
\text { Anti-hypercholesterolemic }(0.877)\end{array}$ & $\begin{array}{c}\text { Respiratory analeptic }(0.987) \\
\text { Anesthetic general }(0.938) \\
\text { Antiprotozoal (Leishmania) }(0.927) \\
\text { Cardiotonic }(0.791) \\
\text { Antithrombotic }(0.557)\end{array}$ \\
\hline 116 & $\begin{array}{c}\text { Chemopreventive }(0.918) \\
\text { Antineoplastic }(0.893) \\
\text { Proliferative diseases treatment }(0.890) \\
\text { Dementia treatment }(0.738)\end{array}$ & $\begin{array}{c}\text { Neuroprotector }(0.983) \\
\text { Anti-hypercholesterolemic }(0.919) \\
\text { Acute neurologic disorders treatment } \\
(0.636) \\
\text { Hypolipemic }(0.626)\end{array}$ & $\begin{array}{c}\text { Respiratory analeptic }(0.989) \\
\text { Anesthetic general }(0.949) \\
\text { Antiprotozoal (Leishmania) (0.936) }\end{array}$ \\
\hline 117 & $\begin{array}{c}\text { Antineoplastic }(0.757) \\
\text { Prostate disorders treatment }(0.678) \\
\text { Proliferative diseases treatment }(0.599)\end{array}$ & $\begin{array}{c}\text { Neuroprotector }(0.852) \\
\text { Anti-hypercholesterolemic }(0.828) \\
\text { Lipid metabolism regulator }(0.732)\end{array}$ & $\begin{array}{c}\text { Respiratory analeptic }(0.853) \\
\text { Erythropoiesis stimulant }(0.851) \\
\text { Immunomodulator (HIV) }(0.829)\end{array}$ \\
\hline 118 & $\begin{array}{c}\text { Antineoplastic }(0.869) \\
\text { Apoptosis agonist }(0.775) \\
\text { Alzheimer's disease treatment }(0.571)\end{array}$ & & $\begin{array}{c}\text { Anti-eczematic }(0.924) \\
\text { Immunosuppressant }(0.790)\end{array}$ \\
\hline 119 & $\begin{array}{c}\text { Antineoplastic }(0.900) \\
\text { Prostate disorders treatment }(0.741)\end{array}$ & & $\begin{array}{c}\text { Anti-eczematic }(0.842) \\
\text { Erythropoiesis stimulant }(0.827)\end{array}$ \\
\hline 120 & $\begin{array}{c}\text { Antineoplastic }(0.869) \\
\text { Apoptosis agonist }(0.775) \\
\text { Alzheimer's disease treatment }(0.671)\end{array}$ & & $\begin{array}{c}\text { Anti-eczematic }(0.924) \\
\text { Immunosuppressant }(0.790) \\
\text { Anti-asthmatic }(0.644)\end{array}$ \\
\hline 121 & $\begin{array}{c}\text { Antineoplastic }(0.863) \\
\text { Apoptosis agonist }(0.724)\end{array}$ & & $\begin{array}{l}\text { Anti-inflammatory }(0.834) \\
\text { Immunosuppressant }(0.798)\end{array}$ \\
\hline 122 & $\begin{array}{c}\text { Antineoplastic }(0.747) \\
\text { Proliferative diseases treatment }(0.641)\end{array}$ & Lipid metabolism regulator $(0.507)$ & $\begin{array}{l}\text { Anti-inflammatory }(0.788) \\
\text { Immunosuppressant }(0.735)\end{array}$ \\
\hline 123 & $\begin{array}{c}\text { Antineoplastic }(0.762) \\
\text { Chemopreventive }(0.643) \\
\text { Proliferative diseases treatment }(0.619)\end{array}$ & $\begin{array}{l}\text { Anti-hypercholesterolemic }(0.671) \\
\text { Cholesterol synthesis inhibitor }(0.543)\end{array}$ & $\begin{array}{l}\text { Anti-eczematic }(0.868) \\
\text { Immunosuppressant }(0.755) \\
\text { Anesthetic general }(0.549)\end{array}$ \\
\hline 124 & $\begin{array}{c}\text { Antineoplastic }(0.882) \\
\text { Prostate disorders treatment }(0.648)\end{array}$ & & $\begin{array}{l}\text { Anti-inflammatory }(0.904) \\
\text { Antiprotozoal }(0.818)\end{array}$ \\
\hline 125 & $\begin{array}{c}\text { Antineoplastic }(0.877) \\
\text { Apoptosis agonist }(0.773) \\
\text { Alzheimer's disease treatment }(0.721)\end{array}$ & $\begin{array}{l}\text { Neurodegenerative diseases treatment } \\
\qquad(0.718)\end{array}$ & $\begin{array}{l}\text { Spasmolytic, urinary }(0.959) \\
\text { Anti-eczematic }(0.924) \\
\text { Immunosuppressant }(0.794)\end{array}$ \\
\hline
\end{tabular}


Table 7. Cont.

\begin{tabular}{|c|c|c|c|}
\hline No. & Antitumor and Related Activity, $(\mathrm{Pa})$ * & Lipid Metabolism Regulators, (Pa) * & Additional Biological Activity, $(\mathrm{Pa})$ * \\
\hline \multirow{2}{*}{126} & Antineoplastic (0.870) & & Immunosuppressant (0.788) \\
\hline & Apoptosis agonist (0.683) & & Growth stimulant $(0.537)$ \\
\hline \multirow{2}{*}{127} & Antineoplastic (skin cancer) (0.650) & & Antibacterial (0.639) \\
\hline & Antineoplastic $(0.650)$ & & Immunomodulator (HIV) (0.568) \\
\hline \multirow{3}{*}{128} & Antineoplastic (0.813) & Hypolipemic $(0.781)$ & Nootropic $(0.610)$ \\
\hline & Apoptosis agonist (0.685) & Anti-hypercholesterolemic (0.594) & Immunosuppressant $(0.601)$ \\
\hline & Chemopreventive (0.542) & Cholesterol synthesis inhibitor $(0.552)$ & \\
\hline \multirow{3}{*}{129} & Apoptosis agonist $(0.844)$ & Hypolipemic $(0.575)$ & Anesthetic (0.689) \\
\hline & Antineoplastic (0.796) & Anti-hypercholesterolemic (0.551) & Anti-inflammatory (0.581) \\
\hline & Chemopreventive (0.631) & Cholesterol synthesis inhibitor (0.507) & \\
\hline \multirow{4}{*}{130} & Antineoplastic (0.858) & Anti-hypercholesterolemic (0.894) & Anti-psoriatic (0.762) \\
\hline & Apoptosis agonist (0.853) & Hypolipemic $(0.762)$ & Anti-eczematic (0.750) \\
\hline & Chemopreventive $(0.840)$ & Lipid metabolism regulator $(0.615)$ & Nootropic $(0.647)$ \\
\hline & Prostate disorders treatment $(0.539)$ & Neuroprotector $(0.611)$ & Anesthetic (0.602) \\
\hline \multirow{3}{*}{131} & Antineoplastic (0.906) & Anti-hypercholesterolemic (0.711) & Immunosuppressant (0.735) \\
\hline & Chemopreventive (0.826) & Lipid metabolism regulator $(0.695)$ & Anti-inflammatory (0.724) \\
\hline & Apoptosis agonist (0.785) & Hypolipemic (0.599) & Urolithiasis treatment $(0.722)$ \\
\hline \multirow{3}{*}{132} & Antineoplastic (0.901) & Anti-hypercholesterolemic (0.770) & Angiogenesis inhibitor $(0.920)$ \\
\hline & Prostate disorders treatment $(0.670)$ & Neuroprotector $(0.700)$ & Respiratory analeptic (0.901) \\
\hline & Apoptosis agonist $(0.627)$ & Hypolipemic $(0.597)$ & Anesthetic general (0.807) \\
\hline
\end{tabular}

*Only activities with $\mathrm{Pa}>0.5$ are shown.

Marine withanolides, paraminabeolides A (120), B (121), C (122), D (123) and F (124) and same compounds named minabeolides 1 (125) and 5 (126) were obtained from lipid extracts of the Formosan soft coral Paraminabea acronocephala. Two compounds (121) and (125) demonstrated cytotoxic toward Hep G2 cancer cells [124]. Twenty years earlier Minabeolides-1 (125) and -5 (126) as C28 steroidal lactones of the withanolide class have been isolated from a soft coral Minabea sp., collected in Truk Lagoon [125].

Secosteroid (127) with epoxide at C-5 and C-6 group from the Formosan soft coral Cespitularia hypotentaculata exhibited cytotoxicity against HT-29 cells [126]. The structures of the steroids (112-132) are shown in Figure 6 and the potential biological activities are shown in Table 5. Two steroids, 11-acetoxy-9,11-secosterols, pinnisterols E (128) and I (129) with a 1,4-quinone moiety, were discovered from the gorgonian coral Pinnigorgia sp. Both identified compounds reduced elastase enzyme release [127]. (22R,23S,24S)-Polyoxygenated steroid named hippuristerone A (130) has been isolated from a Taiwanese gorgonian I. hippuris [128,129]. A rare steroid derivative named griffinipregnone (131) has been isolated from the octocoral Dendronephthya griffin and showed anti-inflammatory activity [130]. An unusual hemiketal steroid, named cladiellin A (132) was isolated from the soft coral Cladiella sp. [131] and a similar steroid 23-keto-cladiellin A (133) was obtained from the monohydroxylated sterol fraction of soft coral Chromonephthea braziliensis [132]. The structures of the steroids (133-156) are shown in Figure 7 and the potential biological activities are shown in Table 8. An unusual pentacyclic hemiacetal sterol nephthoacetal (134) and acetyl derivative (135) were isolated from soft coral Nephthea sp. Compound (134) exhibited a significant inhibitory effect with $\mathrm{EC}_{50}$ value of $2.5 \mu \mathrm{g} / \mathrm{mL}$, while having low toxicity with $\mathrm{LC}_{50}>25.0 \mu \mathrm{g} / \mathrm{mL}$. The in vitro cytotoxic activity of two compounds exhibited moderate cytotoxicity with $\mathrm{IC}_{50}$ values of 12 and $10 \mu \mathrm{g} / \mathrm{mL}$, respectively [133]. 

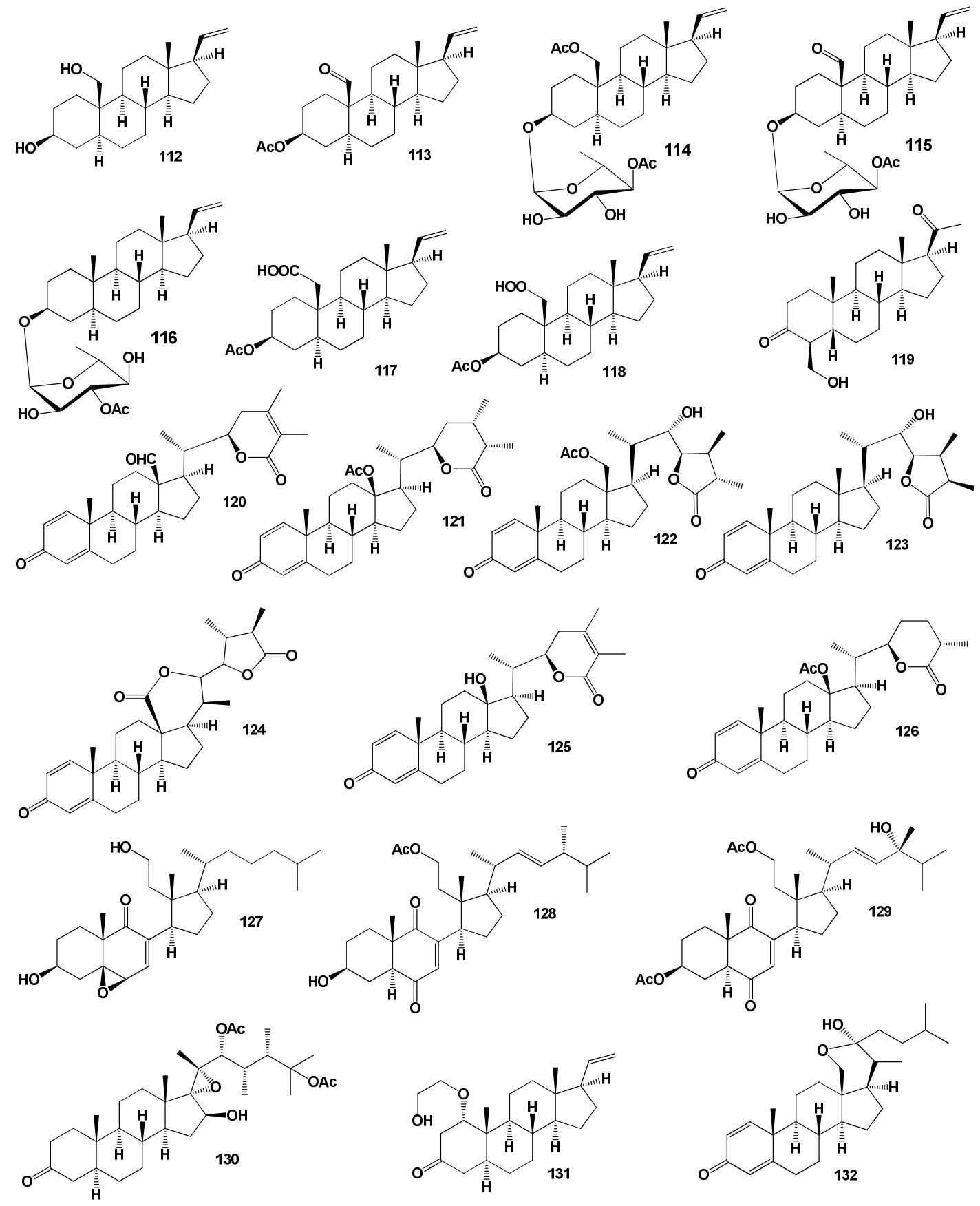

Figure 6. Bioactive steroids derived from soft corals. 

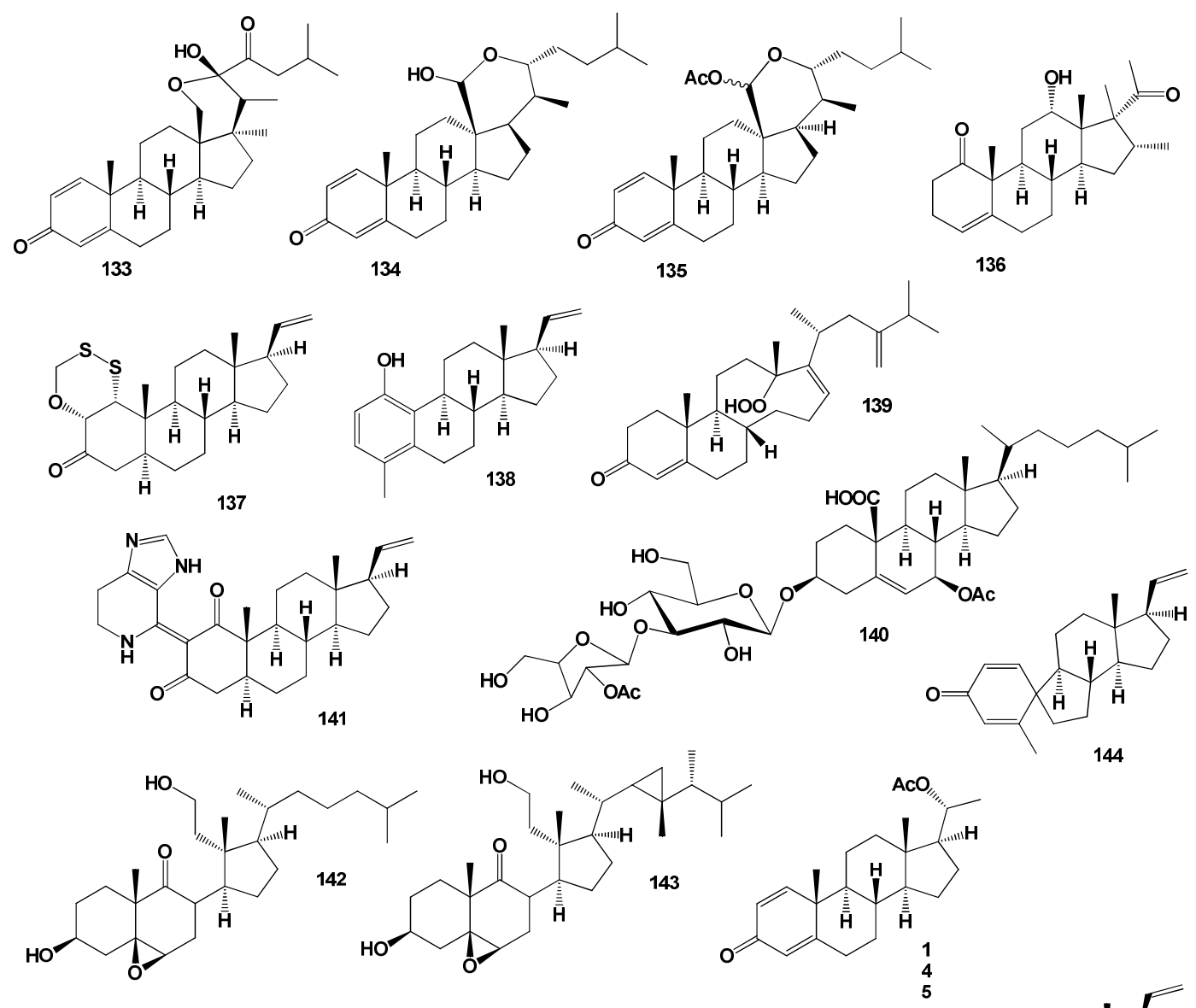

(n)

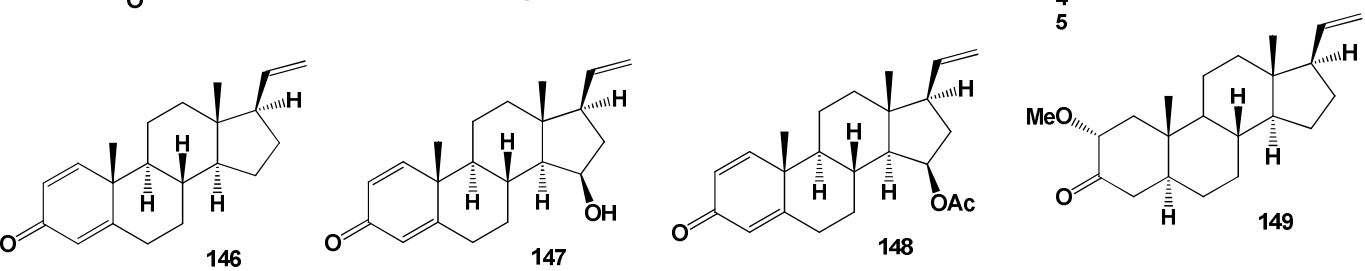

(150)
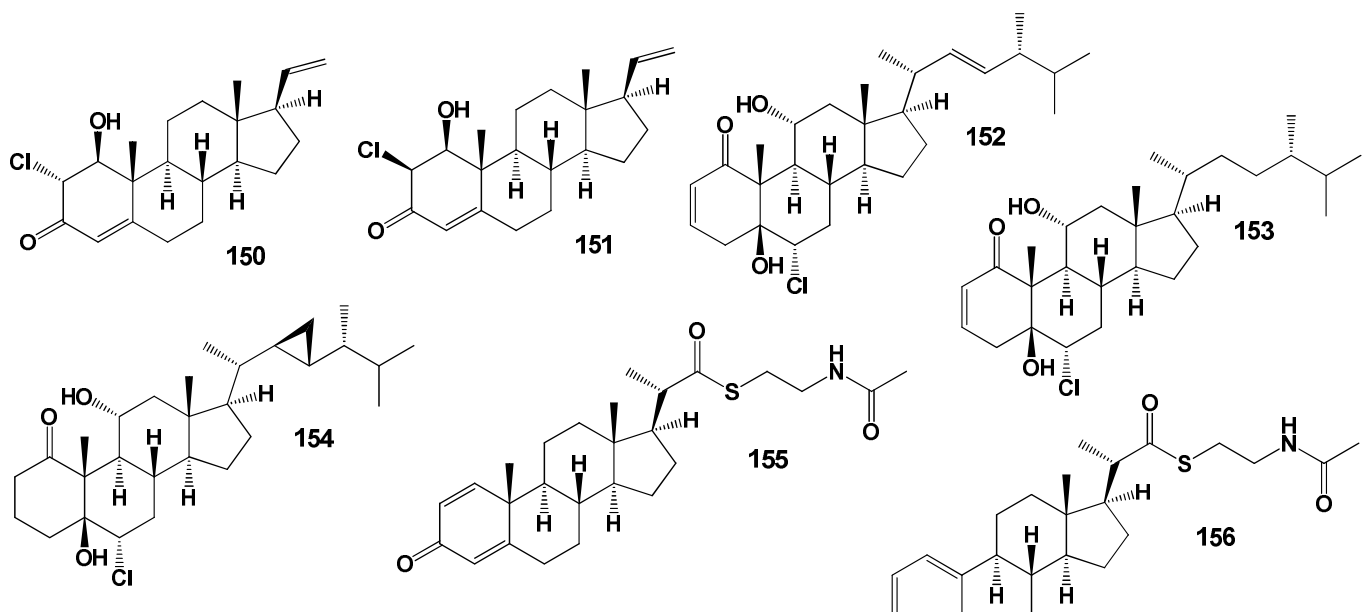

Figure 7. Bioactive steroids derived from soft corals. 
Table 8. Probable biological activities of steroids derived from soft coral species estimated by PASS.

\begin{tabular}{|c|c|c|c|}
\hline No. & Antitumor and Related Activity, (Pa) * & Lipid Metabolism Regulators, $(\mathrm{Pa})^{*}$ & Additional Biological Activity, $(\mathrm{Pa})$ * \\
\hline 133 & $\begin{array}{c}\text { Antineoplastic (0.819) } \\
\text { Prostate disorders treatment }(0.701)\end{array}$ & & $\begin{array}{c}\text { Respiratory analeptic }(0.793) \\
\text { Immunomodulator (HIV) }(0.743)\end{array}$ \\
\hline 135 & Antineoplastic (0.900) & $\begin{array}{c}\text { Neurodegenerative diseases treatment } \\
\qquad(0.519)\end{array}$ & Anti-inflammatory $(0.861)$ \\
\hline 136 & $\begin{array}{c}\text { Antineoplastic }(0.871) \\
\text { Apoptosis agonist }(0.585)\end{array}$ & & $\begin{array}{c}\text { Antiprotozoal (0.763) } \\
\text { Antiprotozoal (Plasmodium) (0.683) }\end{array}$ \\
\hline 137 & $\begin{array}{c}\text { Antineoplastic }(0.880) \\
\text { Proliferative diseases treatment }(0.607)\end{array}$ & & $\begin{array}{c}\text { Antiprotozoal (0.821) } \\
\text { Antiprotozoal (Plasmodium) (0.700) }\end{array}$ \\
\hline 138 & $\begin{array}{l}\text { Antineoplastic }(0.856) \\
\text { Prostate disorders treatment }(0.651) \\
\text { Proliferative diseases treatment }(0.586)\end{array}$ & $\begin{array}{l}\text { Anti-hypercholesterolemic }(0.813) \\
\text { Neuroprotector }(0.734)\end{array}$ & $\begin{array}{c}\text { Anti-inflammatory }(0.929) \\
\text { Immunomodulator }(\mathrm{HIV})(0.868) \\
\text { Anesthetic general }(0.813)\end{array}$ \\
\hline 141 & $\begin{array}{c}\text { Antineoplastic }(0.862) \\
\text { Chemoprotective }(0.694)\end{array}$ & $\begin{array}{c}\text { Hypolipemic ( } 0.554) \\
\text { Cholesterol synthesis inhibitor }(0.509)\end{array}$ & $\begin{array}{l}\text { Immunosuppressant (0.753) } \\
\text { Antiprotozoal (Plasmodium) (0.658) }\end{array}$ \\
\hline 142 & $\begin{array}{l}\text { Chemopreventive }(0.989) \\
\text { Proliferative diseases treatment }(0.969) \\
\text { Antineoplastic }(0.874) \\
\text { Alzheimer's disease treatment }(0.570)\end{array}$ & $\begin{array}{c}\text { Anti-hypercholesterolemic }(0.977) \\
\text { Neuroprotector }(0.895) \\
\text { Atherosclerosis treatment }(0.601) \\
\text { Neurodegenerative diseases treatment } \\
(0.590)\end{array}$ & $\begin{array}{c}\text { Hepatoprotectant }(0.986) \\
\text { Respiratory analeptic }(0.978) \\
\text { Antimycobacterial }(0.939) \\
\text { Antiprotozoal (Leishmania) }(0.772)\end{array}$ \\
\hline 143 & Antineoplastic (0.749) & & $\begin{array}{l}\text { Anti-eczematic }(0.729) \\
\text { Dermatologic }(0.651) \\
\text { Anti-psoriatic }(0.570)\end{array}$ \\
\hline 147 & $\begin{array}{c}\text { Antineoplastic }(0.828) \\
\text { Proliferative diseases treatment }(0.699)\end{array}$ & $\begin{array}{c}\text { Neuroprotector }(0.640) \\
\text { Acute neurologic disorders treatment } \\
(0.626)\end{array}$ & $\begin{array}{l}\text { Anti-inflammatory }(0.920) \\
\text { Respiratory analeptic }(0.838)\end{array}$ \\
\hline 148 & $\begin{array}{l}\text { Antineoplastic }(0.837) \\
\text { Apoptosis agonist }(0.607)\end{array}$ & $\begin{array}{l}\text { Neuroprotector }(0.684) \\
\text { Autoimmune disorders treatment } \\
(0.611)\end{array}$ & $\begin{array}{l}\text { Anti-inflammatory }(0.889) \\
\text { Antiprotozoal (Leishmania) }(0.571)\end{array}$ \\
\hline 149 & $\begin{array}{l}\text { Antineoplastic }(0.851) \\
\text { Proliferative diseases treatment }(0.687)\end{array}$ & $\begin{array}{c}\text { Neuroprotector }(0.771) \\
\text { Acute neurologic disorders treatment } \\
(0.600)\end{array}$ & $\begin{array}{c}\text { Anti-inflammatory (0.923) } \\
\text { Immunomodulator (HIV) }(0.859)\end{array}$ \\
\hline 150 & $\begin{array}{c}\text { Antineoplastic }(0.849) \\
\text { Proliferative diseases treatment }(0.709)\end{array}$ & $\begin{array}{c}\text { Neuroprotector }(0.736) \\
\text { Anti-hypercholesterolemic }(0.634) \\
\text { Acute neurologic disorders treatment } \\
(0.615)\end{array}$ & $\begin{array}{c}\text { Anti-inflammatory (0.910) } \\
\text { Antiprotozoal (Leishmania) (0.584) }\end{array}$ \\
\hline 151 & $\begin{array}{c}\text { Antineoplastic }(0.818) \\
\text { Apoptosis agonist }(0.571)\end{array}$ & & $\begin{array}{l}\text { Respiratory analeptic }(0.858) \\
\text { Cardiotonic }(0.612)\end{array}$ \\
\hline 152 & $\begin{array}{c}\text { Antineoplastic }(0.832) \\
\text { Proliferative diseases treatment }(0.578)\end{array}$ & Anti-hypercholesterolemic (0.789) & $\begin{array}{l}\text { Respiratory analeptic }(0.894) \\
\text { Immunomodulator (HIV) }(0.857)\end{array}$ \\
\hline 153 & $\begin{array}{c}\text { Antineoplastic }(0.837) \\
\text { Proliferative diseases treatment }(0.598)\end{array}$ & Anti-hypercholesterolemic (0.789) & $\begin{array}{c}\text { Respiratory analeptic (0.897) } \\
\text { Immunomodulator (HIV) }(0.858)\end{array}$ \\
\hline 154 & $\begin{array}{l}\text { Apoptosis agonist }(0.862) \\
\text { Antineoplastic }(0.846) \\
\text { Proliferative diseases treatment }(0.623)\end{array}$ & $\begin{array}{l}\text { Anti-hypercholesterolemic }(0.911) \\
\text { Hypolipemic }(0.751) \\
\text { Atherosclerosis treatment }(0.611)\end{array}$ & $\begin{array}{l}\text { Antidiabetic (type 2) (0.617) } \\
\text { Antifungal }(0.584)\end{array}$ \\
\hline
\end{tabular}

* Only activities with $\mathrm{Pa}>0.5$ are shown. 
Extract of the soft coral Dendronephthya gigantea demonstrated the antiproliferative effect against the proliferation of HL-60 human leukemia cells and MCF-7 human breast cancer cells. The steroid 12-hydroxy-16,17-dimethyl-pregn-4-ene-1,20-dione (136) was isolated from the coral sterol fraction [134].

Krempenes A (137) and B (138) are unprecedented pregnane-type steroids that have been isolated from the marine soft coral Cladiella krempfi [135]. Steroid (137) contains a very unusual structural motif, with a hexacyclic oxadithiino unit fused to the steroidal ring A.

A rare steroidal hydroperoxide, 13,14-seco-22-norergosta-4,24(28)-dien-19-hydro-peroxide-3-one (139) has been found in the diethyl ether fraction of the Red Sea soft coral, Litophyton arboretum [136]. Steroid glycoside, dimorphoside B (140) has been isolated from the Western Pacific gorgonian Anthoplexaura dimorpha as the cell-division inhibitors in the sea urchin egg assay [137]. A unique unprecedented spinaceamine-bearing pregnane named scleronine (141) produces a Chinese soft coral Scleronephthya sp. [138].

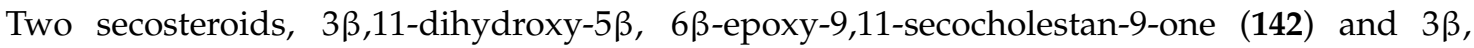
11-dihydroxy-5 $\beta, 6 \beta$-epoxy-9,11-secogorgostan-9-one (143) have been found and identified from extracts of the Taiwanese soft coral Cespitularia taeniata [139-141].

Two steroids (144 and 145) and pregna-1,4,20-trien-3-one (146) have been isolated from the Pacific octocoral Carijoa multiflora. Compound (144) possesses a spiropregnane-based steroidal skeleton and showed antibacterial activity [142]. Compounds (145 and 146) and similar pregnane steroids (147 and 148) have been isolated from a gorgonian Carijoa sp. collected from the South China Sea. Compounds (146, 147 and 148) exhibited cytotoxicity against the human hepatoma cell line Bel-7402, with $\mathrm{IC}_{50}$ values of 9.3, 11.0 and $18.6 \mu \mathrm{M}$, respectively [143]. The Hainan soft coral Scleronephthya gracillimum releases pregnane analogue (149) [144] and two unique chloro-pregnane steroids (150 and 151) have been isolated from the eastern Pacific octocoral Carijoa multiflora [145]. Another three chlorinated marine steroids, yonarasterols G (152), H (153) and I (154), were isolated from the Okinawan soft coral, Clavularia viridis [146].

Unusual steroid thioesters, parathiosteroids A (155) and C (156) were isolated from the 2-propanol extract of the soft coral Paragorgia sp. collected in Madagascar. Both compounds displayed cytotoxicity against a panel of three human tumor cell lines at the micromolar level [147].

The soft coral Lobophytum michaelae that lives on the coast of Taitung and its ethyl acetate extract contained three cytotoxic polyoxygenated steroids called michosterols A-C (157-159) [148] and also ethyl acetate extract the gorgonian Leptogorgia sp. collected from the South China Sea contained dihydroxy-ketosteroid (160) [149]. The structures of the steroids (157-177) are shown in Figure 8 and the potential biological activities are shown in Table 9.

The natural analog of calcitriol (1,25-dihydroxy-vitamin D3, 161) called astrogorgiadiol (162) was first isolated in 1989 by Fusetani and co-workers [150] and another 9,10-secosteroids called calicoferols B (163), D (164) and E (165) have been isolated from an undescribed gorgonian of the genus Muricella [151,152].

Cytotoxic steroidal saponins, astrogorgiosides A (166) bearing acetamido-glucose moiety and astrogorgioside C (167) with a 19-nor and bearing an aromatized B ring steroid aglycone were obtained from the gorgonian Astrogorgia dumbea collected near Dongshan Island in the East China Sea. Both isolated compounds exhibited moderate cytotoxic activity with $\mathrm{IC}_{50}$ values of 26.8 and 45.6 $\mu \mathrm{M}$ against human tumor cells Bel-7402 and K562, respectively [153]. Rare pregnane derivative, 3-dimethoxy-5 $\alpha$-pregnan-20-one (168) has been detected in ethanol extract the Indian ocean gorgonian Subergorgia suberosa, collected from the Mandapam area, Tamil Nadu, India [154].

The cholestane class steroidal hemiacetals named anastomosacetals A (169) and D (170) were obtained from the gorgonian coral Euplexaura anastomosans, collected off the shore of Keomun Island, South Sea Korea [155]. Gorgonian species, Bebryce indica, collected off the coast of Sanya (Hainan, China) was found to contain steroidal glycoside named bebrycoside (171) [156]. Bebrycoside (171) and similar 27-O-[ $\beta$-D-arabino-pyranosyloxy]-20 $\beta, 22 \alpha$-dihydroxy-cholest-4-ene-3-one, named muricellasteroid D (172) and also rare steroid $22 \alpha-\mathrm{O}$-acetyl-2 $\beta$-O-methylene-[4 $\beta$-hydroxy-phenly]-cholest-4-ene-3-one, 
named muricellasteroid $\mathrm{E}$ (173) have been isolated from the EtOH/CH${ }_{2} \mathrm{Cl}_{2}$ extracts of the South China Sea gorgonian coral Muricella flexuosa. Compounds $\mathbf{1 7 2}$ and $\mathbf{1 7 3}$ showed moderate cytotoxicity against A375, K562 and A549 cancer cell lines [157].

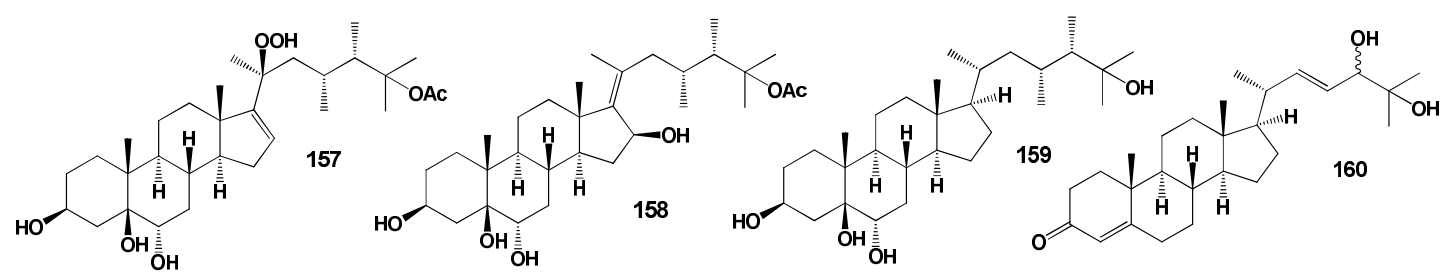

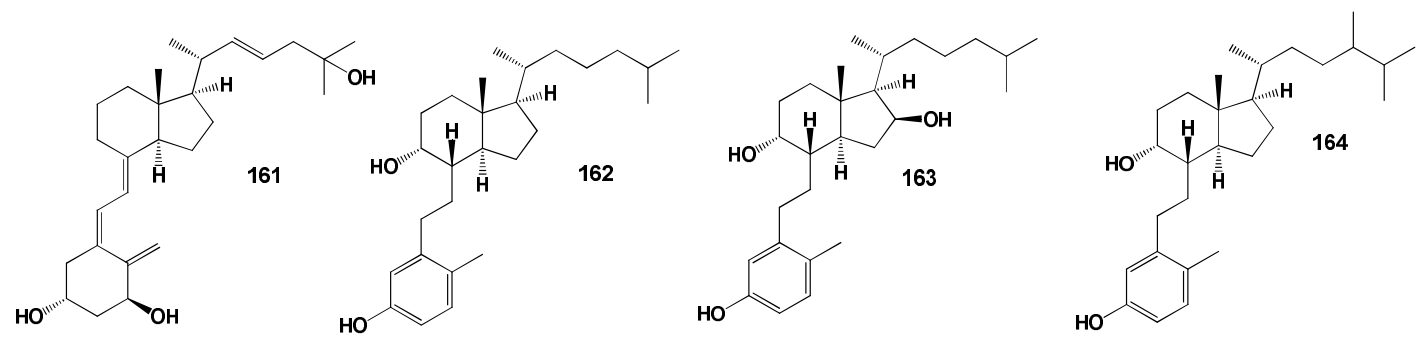

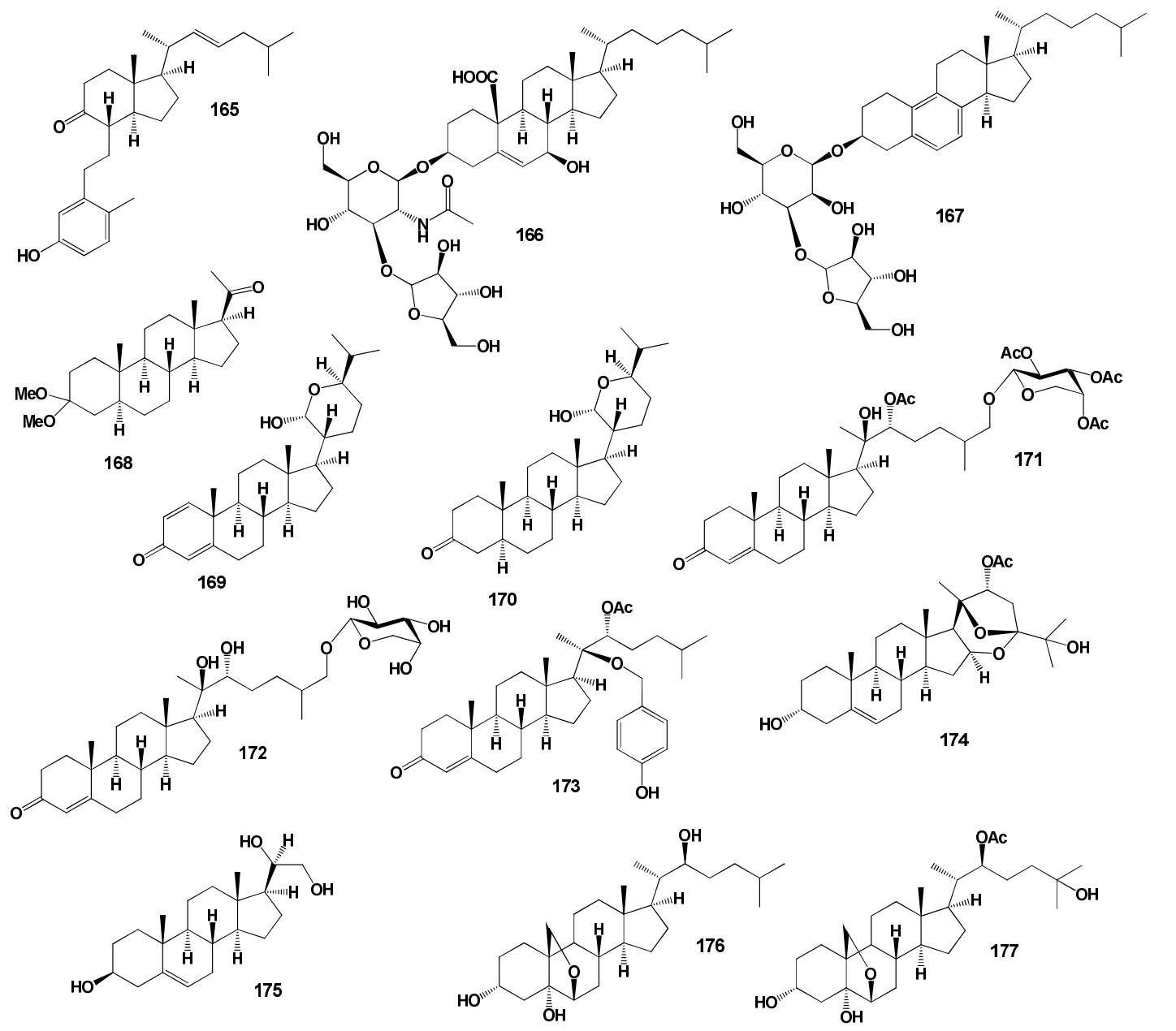

Figure 8. Bioactive steroids derived from soft corals. 
Table 9. Probable biological activities of steroids derived from soft coral species estimated by PASS.

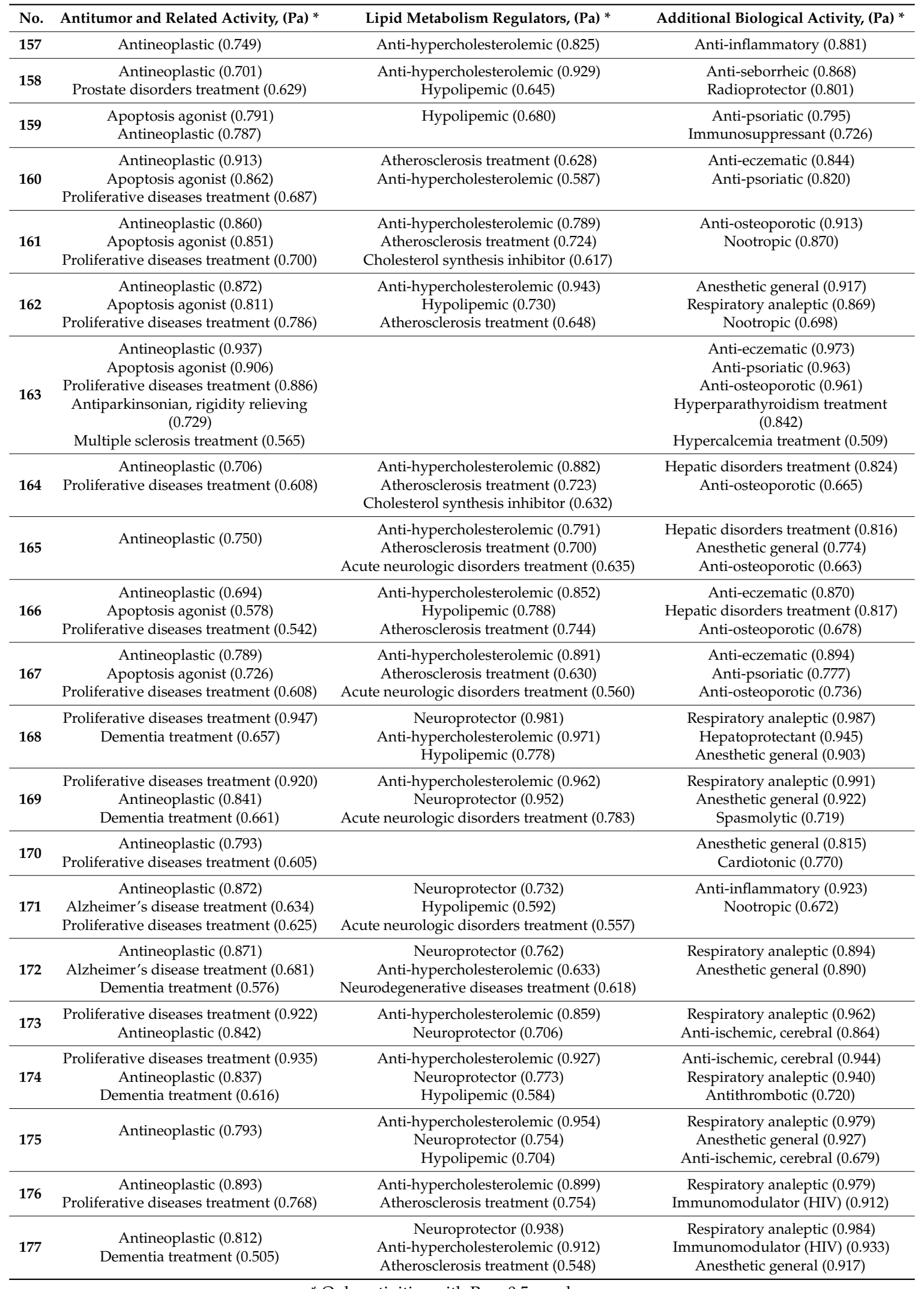

* Only activities with $\mathrm{Pa}>0.5$ are shown.

Rare, a spiroketal steroid, 22-acetoxy-3,25-dihydroxy-16,24,20-24-bisepoxy- $(3 \beta, 16 \alpha, 20 S, 22 R$, 24S)-cholest-5-ene (174) was isolated from the Indian Ocean gorgonian, Gorgonella umbraculum [158] 
and trihydroxy sterol, pregna-5-ene-3 $\beta, 20 \alpha, 21$-triol (175) has been isolated from the Gulf of California gorgonian Muricea cf. austera [159]. Oxysterols (176-181) were detected and identified from an octocoral of the genus Gorgonia from the eastern Pacific of Panama [160]. The structures of the steroids (178-187) are shown in Figure 9 and the potential biological activities are shown in Table 10.

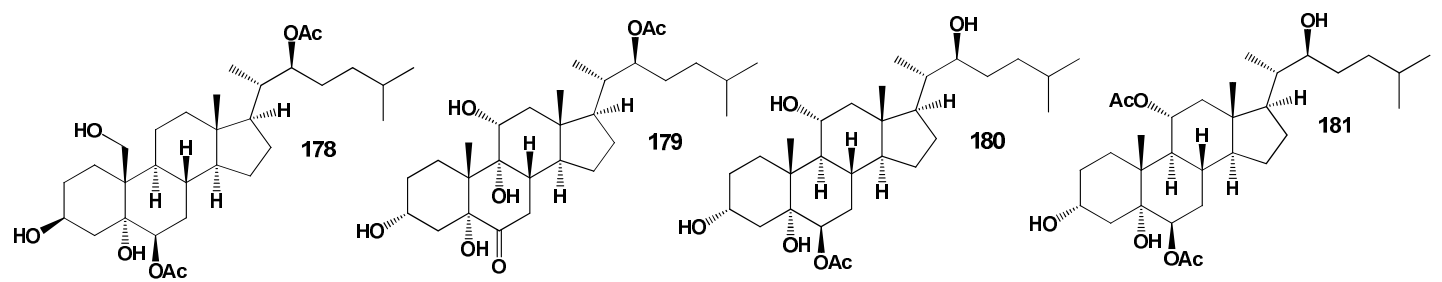

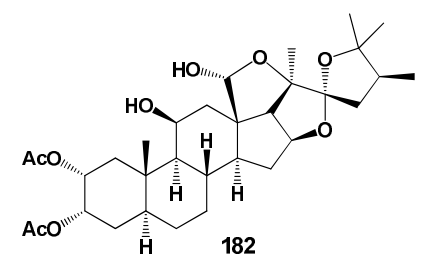

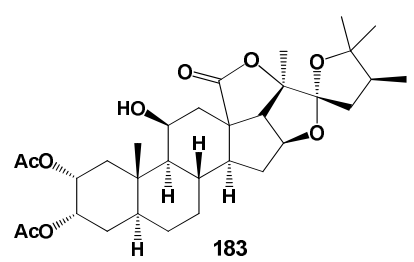

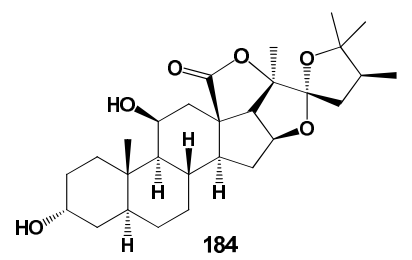

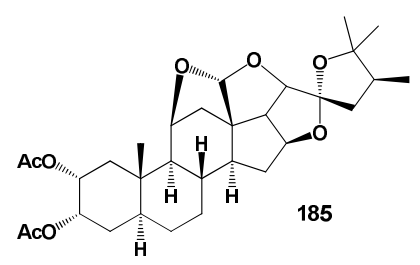

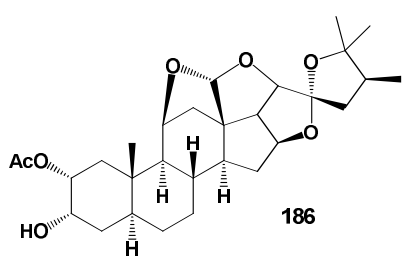

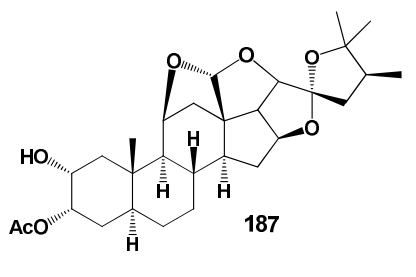

Figure 9. Bioactive steroids derived from soft corals.

Table 10. Probable biological activities of steroids derived from soft coral species estimated by PASS.

\begin{tabular}{|c|c|c|c|}
\hline No. & Antitumor and Related Activity, $(\mathrm{Pa}) *$ & Lipid Metabolism Regulators, $(\mathrm{Pa})$ * & Additional Biological Activity, $(\mathrm{Pa})$ * \\
\hline \multirow{3}{*}{178} & Antineoplastic (0.886) & Anti-hypercholesterolemic (0.716) & Respiratory analeptic (0.966) \\
\hline & Apoptosis agonist $(0.829)$ & Hypolipemic $(0.596)$ & Immunosuppressant (0.837) \\
\hline & Proliferative diseases treatment $(0.710)$ & Atherosclerosis treatment $(0.588)$ & Anesthetic general (0.775) \\
\hline \multirow{2}{*}{179} & Antineoplastic (0.901) & Anti-hypercholesterolemic (0.839) & Respiratory analeptic (0.979) \\
\hline & Proliferative diseases treatment $(0.832)$ & Atherosclerosis treatment $(0.597)$ & Anesthetic general (0.841) \\
\hline \multirow{3}{*}{180} & Antineoplastic (0.897) & \multirow{3}{*}{$\begin{array}{l}\text { Anti-hypercholesterolemic }(0.865) \\
\text { Atherosclerosis treatment }(0.639)\end{array}$} & Respiratory analeptic $(0.974)$ \\
\hline & Apoptosis agonist $(0.858)$ & & Anesthetic $(0.904)$ \\
\hline & Proliferative diseases treatment $(0.778)$ & & Anesthetic general $(0.731)$ \\
\hline \multirow{3}{*}{181} & Antineoplastic (0.842) & \multirow{3}{*}{$\begin{array}{c}\text { Anti-hypercholesterolemic }(0.658) \\
\text { Hypolipemic }(0.633)\end{array}$} & Respiratory analeptic (0.941) \\
\hline & Apoptosis agonist $(0.779)$ & & Anesthetic $(0.842)$ \\
\hline & Proliferative diseases treatment $(0.711)$ & & Cardiotonic $(0.730)$ \\
\hline \multirow{3}{*}{182} & Apoptosis agonist (0.828) & Hypolipemic $(0.636)$ & Anesthetic (0.902) \\
\hline & Antineoplastic $(0.827)$ & Atherosclerosis treatment $(0.630)$ & Antithrombotic (0.573) \\
\hline & Proliferative diseases treatment $(0.536)$ & Cholesterol synthesis inhibitor (0.519) & Spasmolytic $(0.538)$ \\
\hline \multirow{3}{*}{183} & Apoptosis agonist (0.898) & Hypolipemic $(0.660)$ & Anesthetic (0.869) \\
\hline & Antineoplastic (0.852) & Atherosclerosis treatment $(0.618)$ & Immunosuppressant (0.798) \\
\hline & Proliferative diseases treatment $(0.519)$ & Lipid metabolism regulator $(0.535)$ & Anti-inflammatory (0.792) \\
\hline \multirow{3}{*}{184} & Antineoplastic (0.881) & \multirow{3}{*}{$\begin{array}{l}\text { Neuroprotector }(0.603) \\
\text { Hypolipemic }(0.568)\end{array}$} & Respiratory analeptic (0.915) \\
\hline & Apoptosis agonist $(0.797)$ & & Antifungal $(0.828)$ \\
\hline & Proliferative diseases treatment $(0.611)$ & & Antiprotozoal (0.718) \\
\hline \multirow{2}{*}{185} & Antineoplastic $(0.937)$ & & Anti-inflammatory (0.939) \\
\hline & Alzheimer's disease treatment $(0.633)$ & & Antiprotozoal (0.663) \\
\hline \multirow{3}{*}{186} & Antineoplastic (0.934) & & Anti-inflammatory (0.934) \\
\hline & Apoptosis agonist (0.792) & & Anti-asthmatic (0.645) \\
\hline & Antimetastatic (0502) & & Antiprotozoal (Plasmodium) (0.601) \\
\hline \multirow{3}{*}{187} & Antineoplastic (0.934) & & Anti-inflammatory $(0.934)$ \\
\hline & Apoptosis agonist (0.792) & & Anti-asthmatic $(0.645)$ \\
\hline & Antimetastatic (0.502) & & Antiprotozoal (Plasmodium) (0.601) \\
\hline
\end{tabular}

* Only activities with $\mathrm{Pa}>0.5$ are shown. 
The I. hippuris, as well as other species of this genus, belong to the bamboo corals that live in the Central-West Pacific, the Indian Ocean, as well as in the Red Sea [161]. Studies of these corals in the last quarter of a century have shown that they satisfy their metabolic requirements for carbon through the products of photosynthesis [162] and only about $9 \%$ from bacterioplankton. These corals synthesize many metabolites, which are highly oxidized products that are oxidized by their own endogenous oxygen. These include numerous highly oxygenated and spiroketal steroids, many of which demonstrate anticancer activity against many cancer cells [163-170].

Over twenty polyoxygenated steroids, including (22S)- $2 \alpha, 3 \alpha$-diacetoxy-11 $\beta, 18 \beta$-dihydroxy-24-

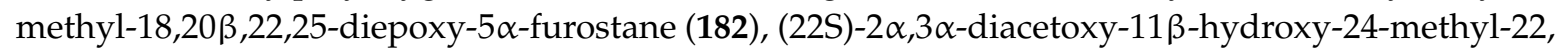
25-epoxy-5 $\alpha$-furostan-18,20 $\beta$-lactone (183) as well as (184-187) were extracted with a mixture of n-hexane and $\mathrm{CH}_{2} \mathrm{Cl}_{2}$ from the I. hippuris living in the southeast coast of Taiwan. Many of the steroids showed cytotoxic activity against Hep G2, Hep 3B, A549, MCF-7 and MDAMB-231 cells [171].

\section{Comparison of Biological Activities of Natural Soft Coral Steroids}

The biological activity of the molecule depends on its structure, which allows analyzing the structure-activity relationships (SAR). This idea was first proposed by Brown and Fraser in 1868 [172] more than 150 years ago; however, it was further developed in the mid-1970s [173,174].

The quantitative structure-activity relationships (QSAR) paradigm was first implemented in toxicology, pharmaceutical and medicinal chemistry, and, ultimately, various aspects of organic and bioorganic chemistry [175]. For over 50 years, the QSAR paradigm has been widely used due to its original postulate that activity was a function of the structure described by electronic attributes, hydrophobicity or steric properties [176]. The rapid and extensive development of methodologies and computational methods led to the definition and refinement of many approaches that introduce the paradigm into the practice of research and development [177].

Several computer programs can estimate with some degree of certainty the pharmacological activities of organic metabolites isolated from natural sources or synthetic compounds [178-180]. Classical (Q)SAR methods are based on the analysis of (quantitative) structure-activity relationships for a single or several biological activities using the compounds belonging to the same chemical series as the training set [181].

Computer program PASS, which is continuously updating and improving for the past thirty years [182], is based on the analysis of a heterogeneous training set included information about more than a million known biologically active compounds with data on ca. 10,000 biological activities [183,184]. Chemical descriptors implemented in PASS, which reflect the peculiarities of ligand-target interactions and original realization of the Bayesian approach for elucidation of structure-activity relationships provide the average accuracy and predictivity for several thousand biological activities equal to about $96 \%[185,186]$. In several comparative studies, it was shown that PASS outperforms in predictivity some other recently developed methods for estimation of biological activity profiles $[187,188]$. Freely available via Internet PASS Online web-service [189] is used by more than twenty thousand researchers from almost a hundred countries to determine the most promising biological activities for both natural and synthetic compounds $[184,186,190-193]$. To reveal the hidden pharmacological potential of the natural substances, we are successfully using PASS for the past fifteen years [194-198].

In the current study, we obtained PASS predictions for two hundred steroids produced by soft corals. PASS estimates are presented as Pa values, which correspond to the probability of belonging to a particular class of "actives" for each predicted biological activity. The higher the Pa value is, the higher is the confidence that the experiment will confirm the predicted biological activity [186].

We have selected about 200 steroids, of which 50 belong to the genus Sinularia, which represent different types of steroids such as secosteroids, spirosteroids, epoxy- and peroxy-steroids, steroid glycosides, halogenated steroids and steroids containing sulfur or nitrogen heteroatoms. The types of steroids presented represent the chemical diversity of these secondary metabolites. Therefore, the pharmacological activities of various types of steroids are of great interest. 
Analyzing the data obtained using PASS, we can state that almost all steroids presented in this article exhibit potential anti-tumor activity with varying degrees of reliability. In addition, forty-one steroids demonstrate anti-tumor and related activity with a confidence level of more than 90 percent, which is of significant interest to the pharmaceutical industry. Figure 10 shows the distribution of steroids with antitumor and related activities in the corals of the genus Sinularia. This group of steroids has a high degree of certainty over 90 percent.

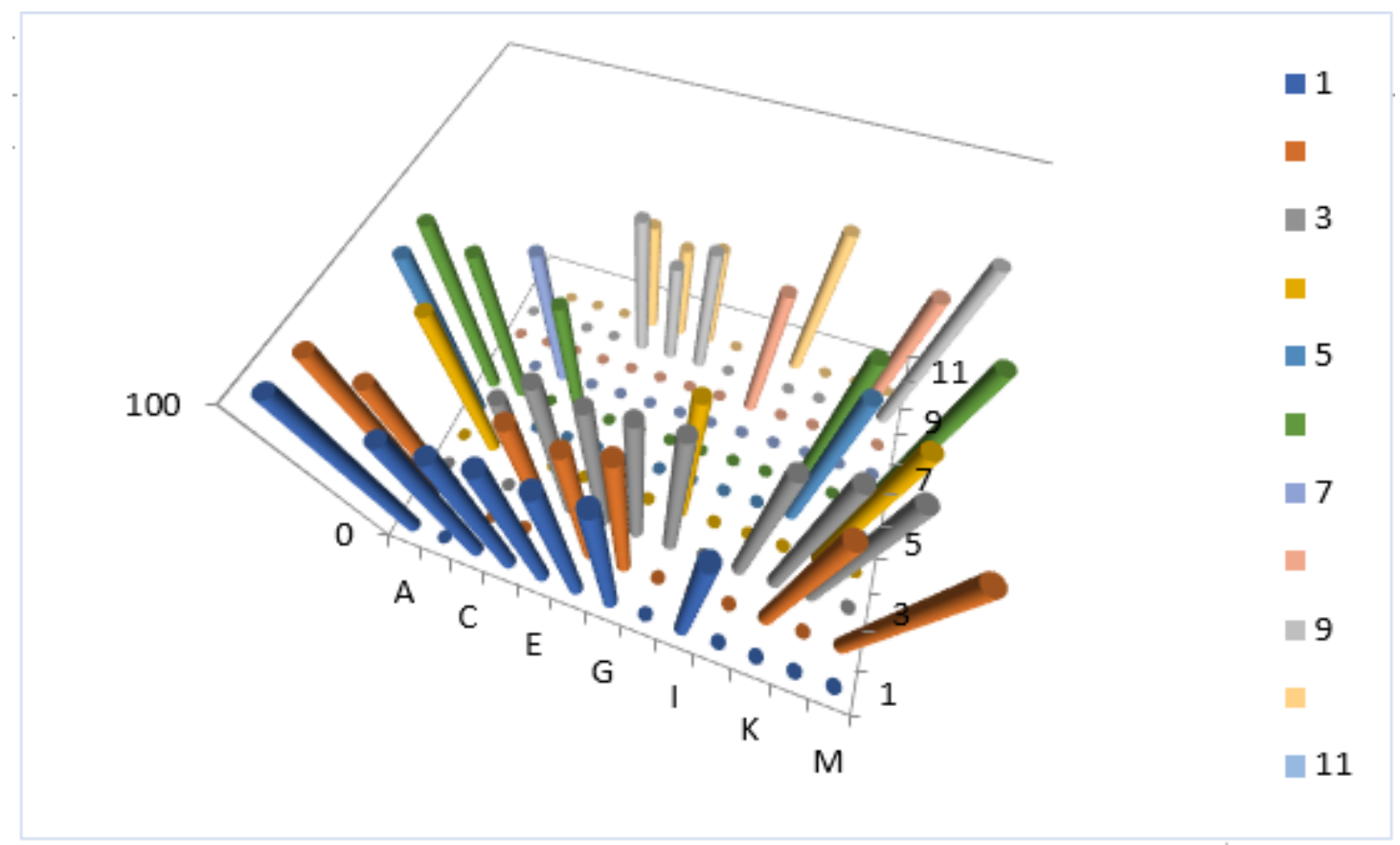

Figure 10. 3D column graph of steroids derived from soft corals of the genus Sinularia that show antitumor and related activities. The letters represent the steroid numbers shown in Figures $1-3$ and Tables 1-3: A-(5), B-(9), C-(18), D-(20), E-(21), F-(22), G-(24), H-(32), I-(37), J-(39), K-(49), $\mathrm{L}-(\mathbf{5 0})$ and $\mathrm{M}-(\mathbf{5 1})$. Steroids that belong to this group, according to the data obtained by the PASS, have confirmed more than 90 percent of their biological activity.

We highlighted other activities in a separate column and named them Lipid metabolism regulators, which include such properties of steroids as anti-hypercholesterolemic, treatment of atherosclerosis, cholesterol synthesis inhibitor and hypolipemic activity. This group includes 32 steroids that show anti-hypercholesterolemic activity with over 90 percent confidence. It is known that hypercholesterolemia and oncogenesis are interrelated, as shown in many studies [199-203]. Therefore, these data are also of great practical interest in identifying the etiology of cancer and its treatment. The column additional activity presents the activities that steroids demonstrate and some activities can also be attributed to the main ones since the reliability of the activities of some steroids exceeds 90 percent of the reliability. For example, the crassarosteroside A (5) water-soluble steroidal glycoside isolated from the Soft Coral S. crassa, according to the authors of the article, demonstrated cytotoxicity against human liver carcinoma (HepG2 and HepG3) [51,52]. Our studies have shown that it demonstrates strong antitumor activity and can be successfully used for the treatment of proliferative diseases, in addition, it demonstrates anti-hypercholesterolemic activity and is also a respiratory analeptic. Figure 11 shows the predicted and calculated (log activities) pharmacological activities of crassarosteroside A.

In addition, some coral steroids show interesting pharmacological activities with a high degree of confidence, over 90\%. For example, the following steroids 15, 32, 76, 138, 149, 150, 184, 185 and 187 show anti-inflammatory activity. And steroids 5, 14, 15, 50, 51, 65, 101, 114, 115, 116, 155, 169, 175, $176,177,178,180$ and 184 can be classified as respiratory analeptic drugs, which are central nervous system stimulants. 

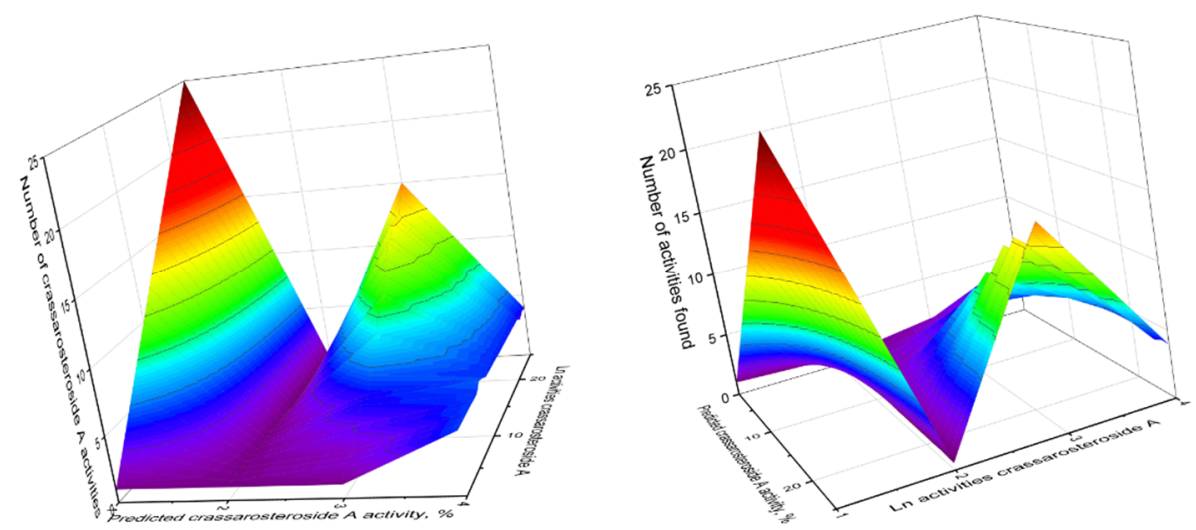

Figure 11. 3D Graph ( $X$ and $Y$ views, predicted and log calculated activities) of the pharmacological activities of water-soluble 3-O-[2'-O-acetyl-( $\alpha$-L-fuco-pryranosyl)]-11,16-hydroxy-24-methylene -cholesterol which called crassarosteroside A (5) was isolated from Sinularia granosa and S. crassa soft coral extracts. According to PASS data, this glycoside demonstrated 25 different activities, with eight activities having a found confidence of more than 90 percent. The main pharmacological activities of crassarosteroside A (5) are respiratory analeptic (99.2\%), proliferative diseases treatment (96.7\%), chemopreventive (95.8\%), anti-hypercholesterolemic (94.7\%), hepatoprotectant $(94.5 \%)$, anticarcinogenic $(90.7 \%)$, antineoplastic $(90.2 \%)$ and anti-inflammatory $(90.0 \%$, Table 1$)$.

Steroids have also been found that exhibit rather rare and extremely specific activities. For example, steroids 18,37 and 38 can be used to treat autoimmune diseases. Steroids 53, 54 and 161 show anti-osteoporotic properties and can be used to treat osteoporosis. Steroids $\mathbf{9 4}$ and 102 are immunomodulators that can be used in the treatment of patients with AIDS. Steroid 163 exhibited strong anti-eczematic and anti-psoriatic properties and steroid $\mathbf{1 2 5}$ exhibited anti-eczematic and spasmolytic properties. Steroid $\mathbf{1 4 2}$ is a hepato-protector, steroid $\mathbf{8 6}$ is an inhibitor of angiogenesis and steroid 87 can be used as a general anesthetic.

\section{Conclusions}

About 200 soft coral steroids are classified as different types of steroids such as secosteroids, spirosteroids, epoxy- and peroxy-steroids, steroid glycosides, halogenated steroids, polyoxygenated steroids and steroids containing sulfur or nitrogen heteroatoms. There also have been found steroids that exhibit rather rare and extremely specific activities. The PASS program is constantly evolving by increasing the database of both natural and synthetic compounds and increasing biological activities by incorporating experimental data. Currently, PASS contains over 1,000,000 chemical structures of natural and synthetic compounds associated with over 10,000 biological activities. These activities are included in the program and are taken from published articles, reviews and other official medical profile documents. Thus, analyzing the presented steroids, it can be stated that more than 40 steroids demonstrate antitumor and related activity with a confidence level of more than 90 percent. Another group, which consists of 32 steroids, demonstrates anti-hypercholesterolemic activity with more than 90 percent confidence.

In addition, ten steroids exhibit anti-inflammatory activity and 20 steroids can be classified as respiratory analeptic drugs. Some steroids with rare structures exhibit anti-osteoporotic properties and can be used to treat osteoporosis, as well as exhibit strong anti-eczemic and anti-psoriatic properties, exhibit anti-eczema and antispasmodic properties. Thus, these data show that soft coral steroids are very interesting in terms of their medical use. However, this requires additional extensive research.

Funding: The study (GTA and PVV) was performed in the framework of the Program for Basic Research of State Academies of Sciences for 2013-2020.

Conflicts of Interest: The authors declare no conflict of interest. 


\section{References}

1. Horvath, E.A. A review of gorgonian coral species (Cnidaria, Octocorallia, Alcyonacea) held in the Santa Barbara museum of natural history research collection: Focus on species from Scleraxonia, Holaxonia, and Calcaxonia-Part I: Introduction, species of Scleraxonia and Holaxonia (Family Acanthogorgiidae). ZooKeys 2019, 860, 1-66.

2. Jankowski, T.; Anokhin, B. Phylum Cnidaria. Thorp. Covichs Freshwater Invertebrat. 2019, 4, 93-111.

3. Boström-Einarsson, L.; Babcock, R.C.; Bayraktarov, E.; Ceccarelli, D.; Cook, N.; Ferse, S.C.A. Coral restorationA systematic review of current methods, successes, failures and future directions. PLoS ONE 2020, 15, e0226631. [CrossRef]

4. Leal, M.C.; Ferrier-Pages, C.; Petersen, D.; Osinga, R. Coral aquaculture: Applying scientific knowledge to ex situ production. Rev. Aquacult. 2014, 6, 1-18.

5. Li, G.; Li, P.; Tang, X. Natural products from corals. In Symbiotic Microbiomes of Coral Reefs Sponges and Corals; Li, Z., Ed.; Springer: Dordrecht, The Netherlands, 2019.

6. Imbs, A.B.; Maliotin, A.N.; Huyen, L.V.; Long, P.Q. Study of fatty acid composition of 17 coral species of Vietnam. Vietnam. J. Sci. Technol. 2005, 43, 84-91.

7. Imbs, A.B.; Demina, O.A.; Demidkova, D.A. Lipid class and fatty acid composition of the boreal soft coral Gersemia rubiformis. Lipids 2006, 41, 721-725. [CrossRef]

8. Imbs, A.B.; Luu, H.V.; Long, P.Q. Lipids of soft coral Sinularia species. Chem. Nat. Comp. 2007, 43, 610-611. [CrossRef]

9. Imbs, A.B.; Dautova, T.N. Use of lipids for chemotaxonomy of octocorals (Cnidaria: Alcyonaria). Russ. J. Mar. Biol. 2008, 34, 205-209. [CrossRef]

10. Imbs, A.B.; Yakovleva, I.M.; Pham, L.Q. Distribution of lipids and fatty acids in the zooxanthellae and host of the soft coral Sinularia sp. Fish. Sci. 2010, 76, 375-380. [CrossRef]

11. Imbs, A.B. Prostaglandins and oxylipins of corals. Russ. J. Mar. Biol. 2011, 37, 325-334. [CrossRef]

12. Imbs, A.B.; Yakovleva, I.M. Dynamics of lipid and fatty acid composition of shallow-water corals under thermal stress: An experimental approach. Coral Reefs 2012, 31, 41-53. [CrossRef]

13. Imbs, A.B. Fatty acids and other lipids of corals: Composition, distribution, and biosynthesis. Russ. J. Mar. Biol. 2013, 39, 153-168. [CrossRef]

14. Elkhawas, Y.A.; Elissawy, A.M.; Elnaggar, M.S.; Mostafa, N.M.; Al-Sayed, E.; Bishr, M.M.; Singa, A.N.B.; Salama, O.M. Chemical diversity in species belonging to soft coral genus Sacrophyton and its impact on biological activity: A review. Mar. Drugs 2020, 18, 41. [CrossRef]

15. Sheu, J.H.; Chen, Y.H.; Chen, Y.-H.; Su, Y.-D.; Chang, Y.-C. Briarane Diterpenoids isolated from gorgonian corals between 2011 and 2013. Mar. Drugs 2014, 12, 2164-2181. [CrossRef]

16. Sikorsky, T.V.; Ermolenko, E.V.; Gloriozova, T.A.; Dembitsky, V.M. Mini Review: Anticancer activity of diterpenoid peroxides. Vietnam J. Chem. 2020, 58, 273-280. [CrossRef]

17. Sunassee, S.N.; Davies-Coleman, M.T. Cytotoxic and antioxidant marine prenylated quinones and hydroquinone. Nat. Prod. Rep. 2012, 29, 513-589. [CrossRef]

18. Hou, X.-M.; Yang, H.; Gu, Y.-C.; Wang, C.-Y.; Shao, C.-L. Chemical and bioactive marine natural products of coral-derived microorganisms (2015-2017). Current Med. Chem. 2019, 26, 6930-6941. [CrossRef]

19. Dembitsky, V.M. Bioactive cyclobutane-containing alkaloids. J. Nat. Med. 2008, 62, 1-33. [CrossRef]

20. Ismail, F.M.D.; Levitsky, D.O.; Dembitsky, V.M. Aziridine alkaloids as potential therapeutic agents. Eur. J. Med. Chem. 2009, 44, 3373-3387. [CrossRef]

21. Dembitsky, V.M. Naturally occurring bioactive cyclobutane-containing (CBC) alkaloids in fungi, fungal endophytes, and plants. Phytomedicine 2014, 21, 1559-1581. [CrossRef]

22. Souza, C.R.M.; Bezerra, W.P.; Sout, J.T. Marine alkaloids with anti-inflammatory activity: Current knowledge and future perspectives. Mar. Drugs 2020, 18, 147. [CrossRef]

23. Xu, J.; Yi, M.; Ding, L.; He, S. A review of anti-inflammatory compounds from marine fungi, 2000-2018. Mar. Drugs 2019, 17, 636. [CrossRef]

24. Dembitsky, V.M. Chemistry and biodiversity of the biologically active natural glycosides. Chem. Biodivers. 2004, 1, 673-781. [CrossRef] [PubMed]

25. Dembitsky, V.M. Astonishing diversity of natural surfactants: 4. Fatty acid amide glycosides, their analogs and derivatives. Lipids 2005, 40, 641-660. [CrossRef] [PubMed] 
26. Dembitsky, V.M. Astonishing diversity of natural surfactants: 5. Biologically active glycosides of aromatic metabolites. Lipids 2005, 40, 869-900. [CrossRef] [PubMed]

27. Dembitsky, V.M. Astonishing diversity of natural surfactants: 6 . Biologically active marine and terrestrial alkaloid glycosides. Lipids 2005, 40, 1081-1114. [CrossRef]

28. Dembitsky, V.M. Astonishing diversity of natural surfactants: 7. Biologically active hemi-and monoterpenoid glycosides. Lipids 2006, 41, 1-27. [CrossRef]

29. Dembitsky, V.M. Astonishing diversity of natural surfactants: 3. Carotenoid glycosides and isoprenoid glycolipids. Lipids 2005, 40, 535-557. [CrossRef]

30. Dembitsky, V.M. Astonishing diversity of natural surfactants: 1 . Glycosides of fatty acids and alcohols. Lipids 2004, 39, 933-953. [CrossRef]

31. Dembitsky, V.M. Astonishing diversity of natural surfactants: 2. Polyether glycosidic ionophores and macrocyclic glycosides. Lipids 2005, 40, 219-248. [CrossRef]

32. Dembitsky, V.M. Antitumor and hepatoprotective activity of natural and synthetic neo steroids. Prog. Lipid Res. 2020, 79, 101048. [CrossRef] [PubMed]

33. Dembitsky, V.M.; Savidov, N.; Gloriozova, T.A. Natural sulphur-containing steroids: Origin and biological activities. Vietnam J. Chem. 2018, 56, 533-541. [CrossRef]

34. Vil, V.A.; Gloriozova, T.A.; Poroikov, V.V.; Terent'ev, A.O.; Savidov, N.; Dembitsky, V.M. Peroxy steroids derived from plant and fungi and their biological activities. Appl. Microbiol. Biotechnol. 2018, 102, 7657-7667. [CrossRef] [PubMed]

35. Dembitsky, V.M.; Gloriozova, T.A.; Poroikov, V.V. Naturally occurring marine $\alpha, \beta$-epoxy steroids: Origin and biological activities. Vietnam J. Chem. 2018, 56, 409-433. [CrossRef]

36. Dembitsky, V.M.; Savidov, N.; Poroikov, V.V.; Gloriozova, T.A.; Imbs, A.B. Naturally occurring aromatic steroids and their biological activities. Appl. Microbiol. Biotechnol. 2018, 102, 4663-4674. [CrossRef]

37. Zhukova, N.V.; Gloriozova, T.A.; Poroikov, V.V.; Dembitsky, V.M. Halogenated (Cl, Br and I) marine steroids and their biological activities: A brief review. Pharma Innov. J. 2017, 6, 456-462.

38. Siddiq, A.; Dembitsky, V. Acetylenic anticancer agents. AntiCancer Agents Med. Chem. 2008, 8, 132-170. [CrossRef]

39. Kuklev, D.V.; Domb, A.J.; Dembitsky, V.M. Bioactive acetylenic metabolites. Phytomedicine 2013, 20, 1145-1159. [CrossRef]

40. Kuklev, D.V.; Dembitsky, V.M. Chemistry, origin, antitumor and other activities of fungal homo-dimeric alkaloids. Mathews J. Pharm. Sci. 2016, 1, 004.

41. Kilimnik, A.; Kuklev, D.V.; Dembitsky, V.M. Antitumor Acetylenic Lipids. Mathews J. Pharmaceut. Sci. 2016, 1,005 .

42. Dembitsky, V.M.; Gloriozova, T.A.; Poroikov, V.V. Novel antitumor agents: Marine sponge alkaloids, their synthetic analogs and derivatives. Mini Rev. Med. Chem. 2005, 5, 319-336. [CrossRef]

43. Wali, A.F.; Majid, S.; Rasool, S.; Shehada, S.B.; Abdulkareem, S.K.; Firdous, A.; Beigh, S.; Shakeel, S.; Mushtaq, S.; Akbar, I.; et al. Natural products against cancer: Review on phytochemicals from marine sources in preventing cancer. Saudi Pharm. J. 2019, 27, 767-777. [CrossRef] [PubMed]

44. Matulja, D.; Wittine, K.; Malatesti, N.; Laclef, S.; Turks, M.; Markovic, M.K.; Ambrožić, G.; Marković, D. Marine natural products with high anticancer activities. Curr. Med. Chem. 2020, 27, 1243-1307. [CrossRef]

45. Di Costanzo, F.; Di Dato, V.; Ianora, A.; Romano, G. Prostaglandins in marine organisms: A review. Mar. Drugs 2019, 17, 428. [CrossRef]

46. McFadden, C.S.; Van Ofwegen, L.P.; Beckman, E.J.; Benayahu, Y.; Alderslade, P. Molecular systematics of the speciose Indo-Pacific soft coral genus, Sinularia (Anthozoa: Octocorallia). Invertebr. Biol. 2009, 128, $303-323$. [CrossRef]

47. Quattrini, A.M.; Wu, T.; Soong, K.; Jeng, M.S.; Benayahu, Y.; McFadden, C.S. A next generation approach to species delimitation reveals the role of hybridization in a cryptic species complex of corals. BMC Evol. Biol. 2019, 19, 116-132. [CrossRef]

48. Lakshmi, V.; Kumar, R. Metabolites from Sinularia species. Nat. Prod. Res. 2009, 23, 801-850. [CrossRef]

49. Chen, W.-T.; Li, Y.; Guo, Y.-W. Terpenoids of Sinularia soft corals: Chemistry and bioactivity. Acta Pharm. Sin. $B$ 2012, 2, 227-237. [CrossRef]

50. Ngoc, N.T.; Huong, P.T.M.; Thanh, N.V.; Cuong, N.X. Steroid Constituents from the Soft Coral Sinularia nanolobata. Chem. Pharm. Bull. 2016, 64, 1417-1419. [CrossRef] 
51. Chao, C.H.; Chou, K.J.; Huang, C.Y.; Wen, Z.H.; Hsu, C.H.; Wu, Y.C.; Dai, C.F.; Sheu, J.H. Steroids from the Soft Coral Sinularia crassa. Mar. Drugs 2012, 10, 439-450. [CrossRef]

52. Mariottini, G.L. The role of cnidaria in drug discovery. In The Cnidaria, Past, Present and Future; Goffredo, S., Dubinsky, Z., Eds.; Springer: Cham, Switzerland, 2016.

53. Ilhan, H.A.; Pulat, C.C. Cytotoxic and antitumor compounds from marine invertebrates. Encycl. Mar. Biotechnol. 2020. [CrossRef]

54. Ngoc, N.T.; Huong, P.T.M.; Van Thanh, N.; Chia, N.T.P.C. Cytotoxic steroids from the Vietnamese soft coral Sinularia conferta. Chem. Pharm. Bull. 2017. [CrossRef]

55. Cường, N.X.; Nhiệm, N.X.; NV Thanh, N.V. Review of chemistry and biological activity studies some marine species in Vietnam in the period 2013-2017. Vietnam J. Chem. 2018, 56, 1-19. [CrossRef]

56. Huang, C.Y.; Su, J.S.; Liaw, C.C.; Sung, P.J.; Chiang, P.L.; Hwang, T.L.; Chang-Feng Dai, J.-H.; Sheu, H. Bioactive steroids with methyl ester group in the side chain from a reef soft coral Sinularia brassica cultured in a tank. Mar. Drugs 2017, 15, 280. [CrossRef]

57. Wu, M.J.; Wang, H.; Jiang, C.S.; Guo, Y.W. New cembrane-type diterpenoids from the South China Sea soft coral Sinularia crassa and their $\alpha$-glucosidase inhibitory activity. Bioorg. Chem. 2020, 104, 104281. [CrossRef]

58. Chen, K.H.; Dai, C.F.; Hwang, T.L.; Chen, C.Y.; Li, J.J.; Chen, J.J. Discovery of novel diterpenoids from Sinularia arborea. Mar. Drugs 2014, 12, 385-393. [CrossRef]

59. Chen, W.T.; Liu, H.L.; Yao, L.G.; Guo, Y.W. 9,11-Secosteroids and polyhydroxylated steroids from two South China Sea soft corals Sarcophyton trocheliophorum and Sinularia flexibilis. Steroids 2014, 92, 56-61. [CrossRef]

60. Ahmed, A.F.; Dai, C.F.; Kuo, Y.H.; Sheu, J.H. $1 \alpha, 3 \beta, 5 \beta$-Trihydroxy-24-methyl-enecholestan-6-one: A novel steroid from a soft coral Sinularia gibberosa. Steroids 2003, 68, 377-381. [CrossRef]

61. Tseng, Y.-J.; Wang, S.K.; Duh, C.Y. Secosteroids and norcembranoids from the soft coral Sinularia nanolobata. Mar. Drugs 2013, 11, 3288-3296. [CrossRef]

62. Thanh, N.V.; Ngoc, N.T.; Anh, H.L.T.; Thung, D.C.; Thao, D.T.; Cuong, N.X. Steroid constituents from the soft coral Sinularia microspiculata. J. Asian Nat. Prod. Res. 2016, 18, 938-944. [CrossRef]

63. Su, J.H.; Tseng, Y.J.; Huang, H.H.; Ahmed, A.F.; Lu, C.K.; Wu, Y.C.; Sheu, J.H. 9,11-Secosterols from the soft corals Sinularia lochmodes and Sinularia leptoclados. J. Nat. Prod. 2006, 69, 850-852. [CrossRef] [PubMed]

64. Yu, S.; Deng, Z.; van Ofwegen, L.; Proksch, P.; Lin, W. 5, 8-Epidioxysterols and related derivatives from a Chinese soft coral Sinularia flexibilis. Steroids 2006, 71, 955-959. [CrossRef] [PubMed]

65. Ngoc, N.T.; Van Thanh, N.; Cuong, N.X.; Nam, N.H. Structure elucidation of four steroids from the soft coral Sinularia nanolobata. Vietnam J. Sci. Technol. 2017, 55, 258-262. [CrossRef]

66. Qia, S.H.; Gao, C.H.; Qian, P.Y.; Zhang, S. Steroids from the South China Sea Gorgonian Subergorgia suberosa. Nat. Prod. Commun. 2010, 5, 201-204. [CrossRef]

67. Kazlauskas, R.; Murphy, P.T.; Ravi, B.N.; Sanders, R.L.; Wells, R.J. Spermidine derivatives and 9,11-secosteroids from a soft coral (Sinularia sp.). Austral. J. Chem. 1982, 35, 69-75. [CrossRef]

68. Bonini, C.; Cooper, C.B.; Kazlauskas, R.; Wells, R.J.; Djerassi, C. Minor and trace sterols in marine invertebrates. 41. Structure and stereochemistry of naturally occurring 9,11-seco sterols. J. Org. Chem. 1983, 48, 2108-2111. [CrossRef]

69. Ciminiello, P.; Fenical, W.; Paul, V.J. Structure assignments of two new C-18-oxygenated steroidal ketals isolated from a pacific soft coral of the genus Sinularia. Experientia 1990, 46, 980-982. [CrossRef]

70. Gebreyesus, T.; Stoilov, I.; Luo, F.T.; Djerassi, C. Minor and trace sterols in marine invertebrates 55. The isolation, structure elucidation and synthesis of ergosta-5,24(28),25-trien-3 $\beta$-ol. Steroids 1985, 45, 447-452. [CrossRef]

71. Jagodzinska, B.M.; Trimmer, J.S.; Fenical, W.; Djerassi, C. Sterols in marine invertebrates. 49. Isolation and structure elucidation of eight new polyhydroxylated sterols from the soft coral Sinularia dissecta. J. Org. Chem. 1985, 50, 1435-1439.

72. Jagodzinska, B.M.; Trimmer, J.S.; Fenical, W.; Djerassi, C. Sterols in marine invertebrates. 51. Isolation and structure elucidation of C-18 functionalized sterols from the soft coral Sinularia dissecta. J. Org. Chem. 1985, 50, 2988-2992.

73. Su, J.; Yu, X.; Zeng, L.; Mak, T.C.W. Noval Polyhydroxylated sterols from the Soft Coral Sinularia numerose. J. Nat. Prod. 1989, 52, 934-940. [CrossRef]

74. Kobayashi, M.; Ishizaka, T.; Miura, N.; Mitsuhashi, H. Marine terpenes and terpenoids. III. Isolation and structures of two cembrane diols from the soft coral Sinularia mayi. Chem. Pharm. Bull. 1987, 35, 2314-2318. 
75. Kobayashi, M.; Krishna, M.M.; Anjaneyulu, V. Marine Sterols. XXIV. Isolation of 24-Methylenecholestane-1 $\alpha$, $3 \beta, 3 \alpha, 6 \beta, 16 \beta$-pentol from Sinularia sp. of soft coral. Chem. Pharm. Bull. 1992, 40, 2845-2846. [CrossRef]

76. Kobayashi, M.; Haribabu, B.; Anjaneyulu, V. Marine Sterol. XXV. Isolation of 23-demethylgorgost-7-ene-3 $\beta$, $5 \alpha, 6 \beta$-triol and(24S)-ergostane-3 $\beta, 5 \alpha, 6 \beta, 7 \beta, 15 \beta$-pentol from soft corals of the Andaman and Nicobar Coasts. Chem. Pharm. Bull. 1993, 41, 87-89. [CrossRef]

77. Anjaneyulu, A.S.R.; Sagar, K.S.; Venugopal, M.J.R.V. Terpenoid and steroid constituents of the Indian Ocean soft coral Sinularia maxima. Tetrahedron 1995, 51, 10997-11010. [CrossRef]

78. Kobayashi, M. Marine sterols. 27. 25-hydroxy derivative of sarcosterol, a novel marine sterol with a 23-methyl and a 17(20)E-double bond, from the soft coral Sinularia mayi. Steroids 1994, 59, 27-29.

79. Ramesh, P.; Venkateswarlu, Y. Novel steroid constituents of the soft coral Sinularia dissecta. Steroids 1999, 64, 785-789. [CrossRef]

80. Xu, S.; Guo, S.; Liu, Y.; Zeng, L. Isolation and identification of two steroids from Sinularia inexplicita. Zhong Yao Cai 2001, 24, 34-35.

81. Weng, J.-R.; Chiu, C.F.; Sheu, J.H. A sterol from soft coral induces apoptosis and autophagy in MCF-7 breast cancer cells. Mar. Drugs 2018, 16, 238. [CrossRef]

82. Chang, Y.C.; Lai, K.H.; Kumar, S.; Chen, P.J.; Wu, Y.H.; Lai, C.L. 1H NMR-Based Isolation of Anti-Inflammatory 9,11-secosteroids from the octocoral Sinularia leptoclados. Mar. Drugs 2020, 18, 271. [CrossRef]

83. Sheu, J.H.; Chang, K.C.; Sung, P.J.; Duh, C.Y. Chemical constituents of a formosan soft coral Sinularia sp. J. Chin. Chem. Soc. 1999, 46, 253-257. [CrossRef]

84. Sheu, J.H.; Chang, K.C.; Duh, C.Y. A Cytotoxic $5 \alpha, 8 \alpha$-epidioxysterol from a soft coral Sinularia species. J. Nat. Prod. 2000, 63, 149-151. [CrossRef]

85. Tillekeratne, L.M.V.; Liyanage, G.K.; Ratnasooriya, W.D.; Ksebati, M.B.; Schmitz, F.J. A new spermatostatic glycoside from the soft coral Sinularia crispa. J. Nat. Prod. 1989, 52, 1143-1145. [CrossRef]

86. Subrahmanyam, C.; Kulatheeswaran, R. Bioactive compounds from a new species of Sinularia soft coral. Indian J. Chem. 1999, 38B, 1388-1390.

87. Blunt, J.W.; Copp, B.R.; Munro, M.H.G.; Northcote, P.T.; Prinsep, M.R. Marine natural products. Nat. Prod. Rep. 2005, 22, 15-61. [CrossRef]

88. Kawagishi, H.; Kawagishi, H.; Choi, J.H.; Choi, J.H.; Ogawa, A.; Ogawa, A.; Yazawa, K. Chaxines B, C, D, and E from the edible mushroom Agrocybe chaxingu. Tetrahedron 2009, 65, 9850-9853.

89. Liu, J.; Wu, X.; Yang, M.; Gu, Y.C.; Yao, L.G.; Huan, X.J.; Miao, Z.H.; Luo, H.; Guo, Y.W. Erectsterates A and $\mathrm{B}$, a pair of novel highly degraded steroid derivatives from the South China Sea soft coral Sinularia erecta. Steroids 2020, 161, 108681. [CrossRef]

90. Jin, P.; Deng, Z.; Pei, Y.; Fu, H.; Lin, W. Polyhydroxylated steroids from the soft coral Sinularia dissecta. Steroids 2005, 70, 487-493. [CrossRef]

91. Zhang, J.; Liang, Y.; Wang, K.L.; Liao, X.J.; Deng, Z.; Xu, S.H. Antifouling steroids from the South China Sea gorgonian coral Subergorgia suberosa. Steroids 2014, 79, 1-6. [CrossRef]

92. Xiao, J.; Gao, M.; Fei, B.; Huang, G.; Diao, Q. Nature-derived anticancer steroids outside cardica glycosides. Fitoterapia 2020, 147, 104757. [CrossRef]

93. Anjaneyulu, A.S.R.; Krishna Murthy, M.V.R.; Gowri, P.M. Novel epoxy steroids from the Indian ocean soft coral Sarcophyton crassocaule. J. Nat. Prod. 2000, 63, 112-118. [CrossRef]

94. Cheng, S.Y.; Dai, C.F.; Duh, C.Y. Sesquiterpenoids and artificial 19-oxygenated steroids from the Formosan soft coral Nephthea erecta. J. Nat. Prod. 2007, 70, 1449-1453. [CrossRef]

95. Wang, S.K.; Puu, S.Y.; Duh, C.Y. Novel steroids from the soft coral Nephthea chabrolii. Tetrahedron 2007, 63, 703-707.

96. Huang, C.Y.; Chang, C.W.; Sheu, J.H. Bioactive steroids from the Formosan soft coral Umbellulifera petasites. Mar. Drugs 2016, 14, 180. [CrossRef]

97. Seo, Y.; Jung, J.H.; Rho, J.R.; Shin, J. Isolation of novel bioactive steroids from the soft coral Alcyonium gracillimum. Tetrahedron 1995, 51, 2497-2506. [CrossRef]

98. Gunatilaka, A.A.L.; Gopichand, Y.; Schmitz, F.J.; Djerassi, C. Minor and trace sterols in marine invertebrates. Isolation and structure elucidation of nine new $5 \alpha, 8 \alpha$-epidioxy sterols from four marine organisms. J. Org. Chem. 1981, 46, 3860-3866. [CrossRef]

99. Tomono, Y.; Hirota, H.; Fusetani, N. Isogosterones A-D, antifouling 13,17-secosteroids from an octocoral Dendronephthya sp. J. Org. Chem. 1999, 64, 2272-2275. [CrossRef] 
100. Chung, H.M.; Hong, P.H.; Su, J.H. Bioactive compounds from a gorgonian coral Echinomuricea sp. (Plexauridae). Mar. Drugs 2012, 10, 1169-1179. [CrossRef]

101. Duh, C.Y.; Lo, I.W.; Wang, S.K.; Dai, C.F. New cytotoxic steroids from the soft coral Clavularia viridis. Steroids 2007, 72, 573-579. [CrossRef]

102. Kobayashi, M.; Lee, N.K.; Byeng, W.; Kazunori, S.; Kyogoku, Y.; Kitagawa, I. Stoloniferone-a, -b, -c, and $-\mathrm{d}$, four new cytotoxic steroids from the Okinawan soft coral Clavularia viridis. Tetrahedron Lett. 1984, 25, 5925-5928. [CrossRef]

103. Kazuo, I.; Makoto, I.; Kinzo, W. Stoloniolide I and II, new marine lactonic steroids with an unprecedented 1,10-secoergostane skeleton, isolated from the Okinawan Soft Coral, Clavularia viridis. Chem. Lett. 1995, 24, 1109-1110.

104. Iwashima, M.; Nara, K.; Iguchi, K. New marine steroids, yonarasterols, isolated from the Okinawan soft coral. Clavularia viridis. Steroids 2000, 65, 130-137. [CrossRef]

105. Chen, W.H.; Wang, S.K.; Duh, C.Y. Polyhydroxylated steroids from the bamboo coral Isis hippuris. Mar. Drugs 2011, 9, 1829-1839. [CrossRef]

106. Cheng, S.-Y.; Dai, C.-F.; Duh, C.-Y. New 4-methylated and 19-oxygenated steroids from the Formosan soft coral Nephthea erecta. Steroids 2007, 72, 653-659. [CrossRef]

107. Amir, F.; Koay, Y.C.; Yam, W.S. Chemical constituents and biological properties of the marine soft coral Nephthea: A review (Part 1). Trop. J. Pharm. Res. 2012, 11, 485-498.

108. Cheng, S.-Y.; Wen, Z.-H.; Wang, S.-K.; Chiang, M.Y.; El Gamal, A.A.H.; Dai, C.-F.; Duh, C.-Y. Revision of the absolute configuration at $\mathrm{C}(23)$ of lanostanoids and isolation of secondary metabolites from Formosan soft coral Nephthea erecta. Chem. Biodiver. 2009, 6, 86-95. [CrossRef]

109. Tsai, Y.Y.; Huang, C.Y.; Tseng, W.R.; Chiang, P.L.; Hwang, T.L.; Su, J.H.; Sung, P.J.; Dai, C.F.; Sheu, J.H. Klyflaccisteroids K-M, bioactive steroidal derivatives from a soft coral Klyxum flaccidum. Bioorg. Med. Chem. Lett. 2017, 27, 1220-1224. [CrossRef]

110. Chang, Y.C.; Kuo, L.M.; Hwang, T.L.; Yeh, J.; Wen, J.H.; Fang, L.S.; Wu, Y.C.; Lin, C.S.; Sheu, J.H.; Sung, P.J. Pinnisterols A-C, new 9,11-secosterols from a Gorgonian Pinnigorgia sp. Mar. Drugs 2016, 14, 12. [CrossRef]

111. Quang, T.H.; Ha, T.T.; Minh, C.V.; Kiem, P.V.; Huong, H.T.; Ngan, N.T.; Nhiem, N.X.; Tung, N.H.; Thao, N.P.; Thuy, D.T. Cytotoxic and PPARs transcriptional activities of sterols from the Vietnamese soft coral Lobophytum laevigatum. Bioorg. Med. Chem. Lett. 2011, 21, 2845-2849. [CrossRef]

112. Chang, Y.-C.; Kuo, L.-M.; Su, J.-H.; Hwang, T.-L.; Kuo, Y.-H.; Lin, C.-S.; Wu, Y.-C.; Sheu, J.-H.; Sung, P.-J. Pinnigorgiols A-C, 9,11-secosterols with a rare ring arrangement from a gorgonian coral Pinnigorgia sp. Tetrahedron 2016, 72, 999-1004. [CrossRef]

113. Chang, Y.C.; Hwang, T.L.; Sheu, J.H.; Wu, Y.C.; Sung, P.J. New anti-inflammatory 9,11-secosterols with a rare tricyclo[5,2,1,1]decane ring from a Formosan Gorgonian Pinnigorgia sp. Mar. Drugs 2016, 14, 218. [CrossRef]

114. Nam, N.H.; Ninh, N.N.; Ngoc, T.T.; Hong, H.S.; Chau, V.M. Cytotoxic steroids from the Vietnamese gorgonian Verrucella corona. Steroids 2018, 138, 57-63. [CrossRef]

115. Chen, W.C.; Sheu, J.H.; Fang, L.S.; Hu, W.P.; Sung, P.J. $3 \alpha, 7 \alpha, 12 \alpha$-Triacetoxy- $5 \beta$-cholanic acid, a steroid from the Formosan soft coral Alcyonium sp. (Alcyoniidae). Nat. Prod. Res. 2006, 20, 748-753. [CrossRef]

116. Abdel-Lateff, A.; Alarif, W.M.; Alburae, N.A.; Algandaby, M.M. Alcyonium octocorals: Potential source of diverse. Bioactive terpenoids. Molecules 2019, 24, 1370. [CrossRef]

117. Häder, D.-P. Natural bioactive compounds technological advancements. In Bioreactive Substances from Coral Reefs and Gorgonians; Sinha, R.P., Häder, D.P., Eds.; Academic Press: Cambridge, MA, USA, 2020; pp. 381-391.

118. Tomono, Y.; Hirota, H.; Imahara, Y.; Fusetani, N. Four new steroids from two octocorals. J. Nat. Prod. 1999, 62, 1538-1541. [CrossRef] [PubMed]

119. Manzo, E.; Ciavatta, M.L.; Nuzzo, G.; Gavagnin, M. Terpenoid content of the Antarctic soft coral Alcyonium antarcticum. Nat. Prod. Commun. 2009, 4, 1615-1619. [CrossRef]

120. Kobayashi, M.; Kiyota, Y.; Orito, S.; Kyogoku, Y.; Kitagawa, I. Five new steroidal glycosides, pregnedioside-A, B, and their three monoacetates, from an Okinawan soft coral of Alcyonium sp. Tetrahedron Lett. 1984, 25, 3731-3734. [CrossRef]

121. Wang, S.K.; Dai, C.F.; Duh, C.Y. Cytotoxic pregnane steroids from the Formosan Soft Coral Stereonephthya crystalliana. J. Nat. Prod. 2006, 69, 103-106. [CrossRef]

122. Fang, H.Y.; Liaw, C.C.; Chao, C.H.; Wen, Z.H.; Wu, Y.C. Bioactive pregnane-type steroids from the soft coral Scleronephthya gracillimum. Tetrahedron 2012, 68, 9694-9700. [CrossRef] 
123. Chen, X.Q.; Xing, N.; Yang, B.; Zhou, X.; Gao, C.; Liu, Y. Two novel sesquiterpenes and a new pregnane derivative from the South China Sea Gorgonian Subergorgia suberosa. Rec. Nat. Prod. 2020, 14, 57-64. [CrossRef]

124. Chao, C.H.; Chou, K.J.; Wen, Z.H.; Wang, G.H.; Wu, Y.C.; Dai, C.F.; Sheu, J.H. Paraminabeolides A-F, cytotoxic and anti-inflammatory marine withanolides from the Soft Coral Paraminabea acronocephala. J. Nat. Prod. 2011, 74, 1132-1141. [CrossRef]

125. Ksebati, M.B.; Schmitz, F.J. Minabeolides: A group of withanolides from a Soft Coral, Minabea sp. J. Org. Chem. 1988, 53, 3926-3929. [CrossRef]

126. Duh, C.-Y.; Li, C.-H.; Wang, S.-K.; Dai, C.-F. Diterpenoids, norditerpenoids, and secosteroids from the Formosan soft coral Cespitularia hypotentaculata. J. Nat. Prod. 2006, 69, 1188-1192. [CrossRef]

127. Chang, Y.-C.; Hwang, T.L.; Sung, P.J. Pinnisterols D-J, new 11-acetoxy-9,11-secosterols with a 1,4-quinone moiety from Formosan Gorgonian coral Pinnigorgia sp. (Gorgoniidae). Mar. Drugs 2017, 15, 11. [CrossRef]

128. Sheu, J.H.; Huang, L.F.; Chen, S.P.; Yang, Y.L. Hippuristerones E-I, new polyoxygenated steroids from the Gorgonian coral Isis hippuris. J. Nat. Prod. 2003, 66, 917-921. [CrossRef]

129. Sheu, J.-H.; Chen, S.P.; Sung, P.J.; Chiang, M.Y.; Dai, C.F. Hippuristerone A, a novel polyoxygenated steroid from the gorgonian Isis hippuris. Tetrahedron Lett. 2000, 41, 7885-7888. [CrossRef]

130. Chao, C.H.; Wen, Z.H.; Su, J.H.; Chen, I.M.; Huang, H.C.; Dai, C.F.; Sheu, J.H. Further study on anti-inflammatory oxygenated steroids from the octocoral Dendronephthya griffini. Steroids 2008, 73, 1353-1358. [CrossRef]

131. Zhang, G.W.; Ma, X.Q.; Kurihara, H.; Zhang, C.X.; Yao, X.S. New hemiketal steroid from the soft coral Cladiella sp. Organic Lett. 2005, 7, 991-994. [CrossRef]

132. Fleury, B.G.; Lages, B.G.; Barbosa, J.P.; Kaiser, C.R.; Pinto, A.C. New hemiketal steroid from the introduced soft coral Chromonephthea braziliensis is a chemical defense against predatory fishes. J. Chem. Ecol. 2008, 34, 987-993. [CrossRef]

133. Zhang, J.; Li, L.-C.; Wang, K.-L.; Liao, X.-J.; Deng, Z.; Xu, S.-H. Pentacyclic hemiacetal sterol with antifouling and cytotoxic activities from the soft coral Nephthea sp. , Bioorg. Med. Chem. Lett. 2013, 23, 1079-1082. [CrossRef]

134. Shanura Fernando, I.P.; Asanka Sanjeewa, K.K.; Kim, H.S.; Wang, L.; Lee, W.W.; Jeon, Y.J. Apoptotic and antiproliferative properties of $3 \beta$-hydroxy- $\Delta 5$-steroidal congeners from a partially purified column fraction of Dendronephthya gigantea against HL-60 and MCF-7 cancer cells. J. Appl. Toxicol. 2017, 11, 1-10.

135. Huang, X.; Deng, Z.; Zhu, X.; van Ofwegen, L.; Proksch, P.; Lin, W. Krempenes A-D: A series of unprecedented pregnane-type steroids from the marine soft coral Cladiella krempfi. Helv. Chim. Acta 2006, 89, 2020-2026. [CrossRef]

136. Ghandourah, M.A.; Alarif, W.M.; Abdel-Lateff, A.; Al-Lihaibi, S.S.; Ayyad, S.-E.N.; Basaif, S.A.; Badria, F.A. Two new terpenoidal derivatives: A himachalene-type sesquiterpene and 13,14-secosteroid from the soft coral Litophyton arboretum. Med. Chem. Res. 2015, 24, 4070-4077. [CrossRef]

137. Fusetani, N.; Yasukawa, K.; Matsunaga, S.; Hashimoto, K. Dimorphosides A and B, novel steroids glycosides from the gorgonian Anthoplexaura dimorpha. Tetrahedron Lett. 1987, 28, 1187-1190. [CrossRef]

138. Cheng, W.; Liu, Z.; Yu, Y.; van Ofwegen, L.; Proksch, P.; Yu, S.; Lin, W. An unusual spinaceamine-bearing pregnane from a soft coral Scleronephthya sp. inhibits the migration of tumor cells. Bioorg. Med. Chem. Lett. 2017, 27, 2736-2741. [CrossRef]

139. Shen, Y.-C.; Cheng, Y.-B.; Kobayashi, J.; Kubota, T.; Takahashi, Y.; Mikami, Y.; Ito, J.; Lin, Y.-S. Nitrogen-containing verticillene diterpenoids from the Taiwanese soft coral Cespitularia taeniata. J. Nat. Prod. 2007, 70, 1961-1965. [CrossRef]

140. Hegazy, M.F.; Mohamed, T.A.; Alhammady, M.A.; Shaheen, A.M.; Reda, E.R.; Elshamy, A.I. Molecular architecture and biomedical leads of terpenes from red sea marine invertebrates. Mar. Drugs 2015, 13, 3154-3181. [CrossRef]

141. Elshamy, A.I.; Nassara, M.I.; Mohamed, T.A.; Hegazy, M.E.F. Chemical and biological profile of Cespitularia species: A mini review. J. Adv. Res. 2016, 7, 209-224. [CrossRef]

142. Díaz-Marrero, A.R.; Porras, G.; Aragón, Z.; de la Rosa, J.M.; Dorta, E.; Cueto, M.; D’Croz, L.; Maté, J.; Darias, J. Carijodienone from the octocoral Carijoa multiflora. A spiropregnane-based steroid. J. Nat. Prod. 2011, 74, 292-295. [CrossRef]

143. Zhao, H.Y.; Shao, C.L.; Li, Z.Y.; Han, L. Bioactive pregnane steroids from a South China Sea Gorgonian Carijoa sp. Molecules 2013, 18, 3458-3466. [CrossRef] 
144. Dorta, E.; Diaz-Marrero, A.R.; Cueto, M.; D’Croz, L.; Maté, J.L.; San-Martín, A.; Darías, J. Two unique chloro-pregnane steroids have been isolated from the eastern Pacific octocoral Carijoa Multiflora. Tetrahedron Lett. 2004, 45, 915-917. [CrossRef]

145. Han, L.; Wang, C.Y.; Huang, H.; Shao, C.L.; Liu, Q.A.; Qi, J.; Sun, X.P.; Zhai, P.; Gu, Y.C. A new pregnane analogue from Hainan soft coral Scleronephthya gracillimum Kukenthal. Biochem. Syst. Ecol. 2010, 38, $243-246$. [CrossRef]

146. Iwashima, M.; Nara, K.; Nakamichi, Y.; Iguchi, K. Three new chlorinated marine steroids, yonarasterols G, H and I, isolated from the Okinawan soft coral, Clavularia viridis. Steroids 2001, 66, 25-32. [CrossRef]

147. Poza, J.; Fernández, R.; Reyes, F.; Reyes, F.; Jiménez, C.; Jiménez, C. Isolation, biological significance, synthesis, and cytotoxic evaluation of new natural parathiosteroids A-C and analogues from the soft coral Paragorgia sp. J. Org. Chem. 2008, 73, 7978-7984. [CrossRef]

148. Huang, C.Y.; Tseng, W.R.; Ahmed, A.F.; Chiang, P.L.; Tai, C.J.; Hwang, T.L.; Dai, C.F.; Sheu, J.H. Anti-inflammatory polyoxygenated steroids from the soft coral Lobophytum michaelae. Mar. Drugs 2018, 16, 93. [CrossRef]

149. Kapustina, I.I.; Makarieva, T.N.; Guzii, A.G.; Kalinovsky, A.I.; Popov, R.S.; Dyshlovoy, S.A.; Grebnev, B.B.; von Amsberg, G.; Stonik, V.A. Leptogorgins A-C, humulane sesquiterpenoids from the Vietnamese gorgonian Leptogorgia sp. Mar. Drugs 2020, 18, 310. [CrossRef]

150. Fusetani, N.; Nagata, H.; Hirota, H.; Tsuyuki, T. Astrogorgiadiol and astrogorgin, inhibitors of cell division in fertilized starfish eggs, from a gorgonian Astrogorgia sp. Tetrahedron Lett. 1989, 30, 7079-7082. [CrossRef]

151. Seo, Y.; Shin, J.; Song, J.I. New secosteroids from an undescribed gorgonian of the genus Muricella. J. Nat. Prod. 1995, 58, 1291-1295. [CrossRef]

152. Masamitsu, O.; Koji, Y.; Hiyoshizo, K.; Kozo, S. Calicoferols A and B, two novel secosterols possessing brine-shrimp lethality from the gorgonian Calicogorgia sp. Chem. Lett. 1991, 20, 427-430.

153. Lu, Y.-N.; Ping, C.; Tian, X.-Q.; Lou, L.-G.; Fan, C.-Q. Unusual cytotoxic steroidal saponins from the gorgonian Astrogorgia dumbea. Planta Med. 2016, 82, 882-887. [CrossRef]

154. Subrahmanyam, C.; Kumar, S.R.; Reddy, G.D. A new pregnane derivative from the Indian ocean gorgonian Subergorgia suberosa (Pallas). Indian J. Chem. 2003, 42, 219-220. [CrossRef]

155. Seo, Y.; Rho, J.-R.; Cho, K.W.; Shin, J. Isolation of new steroidal hemiacetals from the gorgonian Euplexaura anastomosans. J. Nat. Prod. 1996, 59, 1196-1199. [CrossRef]

156. Yang, J.; Qi, S.-H.; Zhang, S.; Xiao, Z.-H.; Li, Q.-X. Bebrycoside, a new steroidal glycoside from the Chinese gorgonian coral Bebryce indica. Pharmazie 2007, 62, 154-155. [CrossRef]

157. Wang, P.; Qi, S.H.; Liu, K.S.; Huang, L.S.; He, F.; Wang, Y.F. Steroids from the South China sea gorgonian coral Muricella Flexuosa. Z. Naturforsch. 2011, 66, 635-640. [CrossRef]

158. Anjaneyulu, A.S.R.; Rao, V.L.; Sastry, V.G. A new spiroketal steroid from gorgonella Umbraculum. Nat. Prod. Res. 2003, 17, 149-152. [CrossRef]

159. Murillo-Alvarez, J.; Encarnacion-Dimayuga, R. New bioactive pregnadiene-derived glycosides from the gulf of California gorgonian Muricea cf. Austera. Pharm. Biol. 2003, 41, 531-535. [CrossRef]

160. Cardoso-Martínez, F.; de la Rosa, J.M.; Díaz-Marrero, A.R.; Darias, J.; D’Croz, L.; Jiménez-Antón, M.D.; Corral, M.J.; Cueto, M. Oxysterols from an octocoral of the genus Gorgonia from the eastern Pacific of Panama. J. RSC Adv. 2013, 6, 1-15. [CrossRef]

161. Fabricius, K.; Alderslade, P. Soft Corals and Sea Fans: A Comprehensive Guide to the Tropical Shallow-Water Genera of the Central-West Pacific, the Indian Ocean and the Red Sea; AIMS: Townsville, Australia, 2001; p. 264.

162. Sorokin, Y.I. Biomass, metabolic rates and feeding of some common zoantharians and octocorals. Aust. J. Mar. Freshw. Res. 1991, 42, 729-741. [CrossRef]

163. Chao, C.H.; Huang, L.F.; Wu, S.L.; Su, J.H.; Huang, H.C.; Sheu, J.H. Steroids from the gorgonian Isis Hippuris. J. Nat. Prod. 2005, 68, 1366-1370. [CrossRef]

164. Chen, W.H.; Wang, S.K.; Duh, C.Y. Polyhydroxylated steroids from the bamboo coral Isis Hippuris. Tetrahedron 2002, 58, 6259-6266. [CrossRef]

165. Sheu, J.H.; Chao, C.H.; Wang, G.H.; Hung, K.C.; Duh, C.Y. The first A-nor-hippuristanol and two novel 4,5-secosuberosanoids from the gorgonian Isis Hippuris. Tetrahedron Lett. 2004, 45, 6413-6416. [CrossRef]

166. Rao, C.B.; Ramana, K.V.; Venkata Rao, D.; Fahy, E.; Faulkner, D.J. Metabolites of the Gorgonian Isis hippuris from India. J. Nat. Prod. 1988, 51, 954-958. [CrossRef]

167. Anisatusholihah, A. The Effects of Gorgonian Isis hippuris on Histologic Grade Score of Adenocarcinoma Mammae Tissue in C3H Mice Strain. Ph.D. Dissertation, Diponegoro University, Semarang, Indonesia, 2009. 
168. Liang, C.H.; Chou, T.H.; Yang, C.C.; Hung, W.J.; Chan, L.C.; Cheng, D.L.; Wang, G.H. Cytotoxic effect of Discosoma sp., Isis hippuris and Nephthea chabrolii on human oral SCC25 cells. J. Taiwan Inst. Chem. Eng. 2010, 41, 333-337. [CrossRef]

169. Putra, M.Y.; Wibowo, J.T.; Murniasih, T. A review of chemistry and biological activities of the Indonesian Octocorallia. J. Appl. Pharm. Sci. 2017, 7, 219-227.

170. Sayuti, M.; Rukmi, W.D.; Yunianta, P. Secondary metabolites and antioxidant potentials of axis sea Bamboo (Isis hippuris). Dhaka Univ. J. Pharm. Sci. 2019, 18, 13-20. [CrossRef]

171. Chao, C.H.; Huang, L.F.; Yang, Y.L.; Su, J.H.; Wang, G.H.; Chiang, M.Y.; Wu, Y.C.; Dai, C.F.; Sheu, J.H. Polyoxygenated steroids from the Gorgonian Isis hippuris. J. Nat. Prod. 2005, 68, 880-885. [CrossRef]

172. Brown, A.C.; Fraser, T.R. The connection of chemical constitution and physiological action. Trans. Roy. Soc. Edinburg 1868, 25, 224-242.

173. Parascandola, J. The controversy over structure-activity relationships in the early twentieth century. Pharm. Hist. 1974, 16, 54-63.

174. Barlow, R.B. Structure-activity relationships. Trends Pharm. Sci. 1979, 1, 109-111. [CrossRef]

175. Hansch, C.; Leo, A. Substituent Constants for Correlation Analysis in Chemistry and Biology; John Wiley \& Sons: New York, NY, USA, 1979.

176. Schultz, T.W.; Cronin, M.T.D.; Walker, J.D.; Aptula, A.O. Quantitative structure-activity relationships (QSARs) in toxicology: A historical perspective. J. Mol. Struct. Theochem. 2003, 622, 1-22. [CrossRef]

177. Allen, T.E.H.; Goodman, J.M.; Gutsell, S.; Russell, P.J. Quantitative predictions for molecular initiating events using three-dimensional quantitative structure-activity relationships. Chem. Res. Toxicol. 2020, 33, 324-333. [CrossRef]

178. Sliwoski, G.; Kothiwale, S.; Meiler, J.; Lowe, E.W., Jr. Computational methods in drug discovery. Pharm. Rev. 2014, 66, 334-395. [CrossRef]

179. Leelananda, S.P.; Lindert, S. Computational methods in drug discovery. Beilstein J. Org. Chem. 2016, 12, 2694-2718. [CrossRef]

180. Kokh, D.B.; Amaral, M.; Bomke, J.; Grädler, U.; Musil, D. Estimation of drug-target residence times by $\tau$-random acceleration molecular dynamics simulations. J. Chem. Theor. Comput. 2018, 14, 3859-3869. [CrossRef]

181. Cherkasov, A.M.; Muratov, E.N.; Fourches, D.; Varnek, A.; Baskin, I.I.; Cronin, M.; Dearden, J. QSAR modeling: Where have you been? Where are you going to? J. Med. Chem. 2014, 57, 4977-5010. [CrossRef]

182. Burov, Y.V.; Poroikov, V.V.; Korolchenko, L.V. National system for registration and biological testing of chemical compounds: Facilities for new drugs search. Bull. Natl. Center Biol. Active Comp. 1990, 1, 4-25.

183. Muratov, E.N.; Bajorath, J.; Sheridan, R.P.; Tetko, I.; Filimonov, D.; Poroikov, V.; Oprea, T. QSAR without Borders. Chem. Soc. Rev. 2020, 49, 3525-3564. [CrossRef]

184. Poroikov, V.V.; Filimonov, D.A.; Gloriozova, T.A.; Lagunin, A.A.; Druzhilovskiy, D.S.; Rudik, A.V. Computer-aided prediction of biological activity spectra for organic compounds: The possibilities and limitations. Russ. Chem. Bull. 2019, 68, 2143-2154. [CrossRef]

185. Filimonov, D.A.; Druzhilovskiy, D.S.; Lagunin, A.A.; Gloriozova, T.A.; Rudik, A.V.; Dmitriev, A.V.; Pogodin, P.V.; Poroikov, V.V. Computer aided prediction of biological activity spectra for chemical compounds: Opportunities and limitations. Biom. Chem. Res. Method 2018, 1, e00004. [CrossRef]

186. Filimonov, D.A.; Lagunin, A.A.; Gloriozova, T.A.; Rudik, A.V.; Druzhilovskiy, D.S.; Pogodin, P.V.; Poroikov, V.V. Prediction of the biological activity spectra of organic compounds using the PASS online web resource. Chem. Heterocycl. Compd. 2014, 50, 444-457. [CrossRef]

187. Anusevicius, K.; Mickevicius, V.; Stasevych, M.; Zvarych, V.; Komarovska-Porokhnyavets, O.; Novikov, V.; Tarasova, O.; Gloriozova, T.; Poroikov, V. Design, synthesis, in vitro antimicrobial activity evaluation and computational studies of new N-(4-iodophenyl)- $\beta$-alanine derivatives. Res. Chem. Intermed. 2015, 41, 7517-7540. [CrossRef]

188. Druzhilovskiy, D.S.; Rudik, A.V.; Filimonov, D.A.; Lagunin, A.A.; Gloriozova, T.A.; Poroikov, V.V. Online resources for the prediction of biological activity of organic compounds. Rus. Chem. Bull. 2016, 65, 384-393. [CrossRef]

189. PASS Online URL. Available online: http://www.way2drug.com/passonline/ (accessed on 20 October 2020).

190. Lagunin, A.A.; Goel, R.K.; Gawande, D.Y.; Priynka, P.; Gloriozova, T.A.; Dmitriev, A.V.; Ivanov, S.M.; Rudik, A.V.; Konova, V.I.; Pogodin, P.V.; et al. Chemo- and bioinformatics resources for in silico drug discovery from medicinal plants beyond their traditional use: A critical review. Nat. Prod. Rep. 2014, 31, 1585-1611. [CrossRef] 
191. Savidov, N.; Gloriozova, T.A.; Poroikov, V.V.; Dembitsky, V.M. Highly oxygenated isoprenoid lipids derived from fungi and fungal endophytes: Origin and biological activities. Steroids 2018, 140, 114-124. [CrossRef]

192. Gawande, D.Y.; Druzhilovsky, D.; Gupta, R.C.; Poroikov, V.; Goel, R.K. Anticonvulsant activity and acute neurotoxic profile of Achyranthes aspera Linn. J. Ethnopharmacol. 2017, 202, 97-102. [CrossRef]

193. Goel, R.K.; Poroikov, V.; Gawande, D.; Lagunin, A.; Randhawa, P.; Mishra, A. Revealing medicinal plants useful for comprehensive management of epilepsy and associated co-morbidities through in silico mining of their phytochemical diversity. Planta Med. 2015, 81, 495-506.

194. Vil, V.; Terent'ev, A.O.; Al Quntar, A.A.A.; Gloriozova, T.A.; Savidov, N.; Dembitsky, V.M. Oxetane-containing metabolites: Origin, structures and biological activities. Appl. Microbiol. Biotechnol. 2019, 103, 2449-2467. [CrossRef]

195. Dembitsky, V.M.; Dzhemileva, L.; Gloriozova, T.; D'yakonov, V. Natural and synthetic drugs used for the treatment of the dementia. Biochem. Biophys. Res. Commun. 2020, 523, 772-783. [CrossRef]

196. Dembitsky, V.M.; Gloriozova, T.A.; Poroikov, V.V. Naturally occurring isocyano/isothiocyanato compounds: Their pharmacological and SAR activities. Mathews J. Pharm. Sci. 2016, 1, 003.

197. Dembitsky, V.M.; Gloriozova, T.A.; Poroikov, V.V. Biological activities of organometalloid (As, At, B, Ge, Si, Se, Te) steroids. J. Appl. Pharm. Sci. 2017, 7, 184-202.

198. Dembitsky, V.M.; Gloriozova, T.A.; Poroikov, V.V. Pharmacological profile of natural and synthetic compounds with rigid adamantane-based scaffolds as potential agents for the treatment of neurodegenerative diseases. Biochem. Biophys. Res. Commun. 2020, 529, 1225-1241. [CrossRef]

199. Herbey, I.I.; Ivankova, N.V.; Katkoori, V.R.; Mamaeva, O.A. Colorectal cancer and hypercholesterolemia: Review of current research. Exp. Oncol. 2005, 27, 166-178.

200. Papanagnou, P.; Stivarou, T.; Papageorgiou, I.; Papadopoulos, G.E.; Pappas, A. Marketed drugs used for the management of hypercholesterolemia as anticancer armament. Onco Targets Ther. 2017, 10, 4393-4411. [CrossRef]

201. Levitsky, D.O.; Dembitsky, V.M. Anti-breast cancer agents derived from plants. Nat. Prod. Bioprospect. 2015, 5, 1-16. [CrossRef]

202. Cho, J.H.; Weinstein, D.A.; Lee, Y.M. Emerging roles of autophagy in hepatic tumorigenesis and therapeutic strategies in glycogen storage disease type IA: A review. J. Inherit. Metab. 2020. [CrossRef]

203. De Cicco, P.; Catani, M.V.; Gasperi, V.; Sibilano, M.; Quaglietta, M.; Savini, I. Nutrition and breast cancer: A literature review on prevention, treatment and recurrence. Nutrients 2019, 11, 1514. [CrossRef]

Publisher's Note: MDPI stays neutral with regard to jurisdictional claims in published maps and institutional affiliations.

(C) 2020 by the authors. Licensee MDPI, Basel, Switzerland. This article is an open access article distributed under the terms and conditions of the Creative Commons Attribution (CC BY) license (http://creativecommons.org/licenses/by/4.0/). 